ApJ 756, 187 (2012)

\title{
The optically unbiased GRB host (TOUGH) survey. I. Survey design and catalogs ${ }^{1}$
}

\author{
Jens Hjorth ${ }^{2}$, Daniele Malesani² ${ }^{2}$ Páll Jakobsson ${ }^{3}$, Andreas O. Jaunsen ${ }^{4}$, \\ Johan P. U. Fynbo ${ }^{2}$, Javier Gorosabel ${ }^{5}$, Thomas Krühler ${ }^{2}$, Andrew J. Levan ${ }^{6}$, \\ Michał J. Michałowski ${ }^{7}$, Bo Milvang-Jensen ${ }^{2}$, Palle Møller ${ }^{8}$, Steve Schulze ${ }^{3}$, \\ Nial R. Tanvir ${ }^{9}$, and Darach Watson ${ }^{2}$
}

\begin{abstract}
Long-duration gamma-ray bursts (GRBs) are powerful tracers of star-forming galaxies. We have defined a homogeneous subsample of 69 Swift GRB-selected galaxies spanning a very wide redshift range. Special attention has been devoted to making the sample optically unbiased through simple and well-defined selection criteria based on the high-energy properties of the bursts and their positions on the sky. Thanks to our extensive follow-up observations, this sample has now achieved a comparatively high degree of redshift completeness, and thus provides a legacy sample, useful for statistical studies of GRBs and their host galaxies. In this paper we present the survey design and summarize the results of our observing program conducted at the ESO Very Large Telescope (VLT) aimed at obtaining the most basic properties of galaxies in this sample, including a catalog
\end{abstract}

\footnotetext{
${ }^{2}$ Dark Cosmology Centre, Niels Bohr Institute, University of Copenhagen, Juliane Maries Vej 30, DK-2100 Copenhagen Ø, Denmark; jens@dark-cosmology.dk

${ }^{3}$ Centre for Astrophysics and Cosmology, Science Institute, University of Iceland, Dunhagi 3, 107 Reykjavík, Iceland

${ }^{4}$ Institute of Theoretical Astrophysics, University of Oslo, PO Box 1029 Blindern, NO-0315 Oslo, Norway

${ }^{5}$ Instituto de Astrofísica de Andalucía (IAA-CSIC), P.O. Box 03004, E-18080 Granada, Spain

${ }^{6}$ Department of Physics, University of Warwick, Coventry CV4 7AL, UK

${ }^{7}$ SUPA, Institute for Astronomy, University of Edinburgh, Royal Observatory, Edinburgh, EH9 3HJ, UK

${ }^{8}$ European Southern Observatory, Karl-Schwarzschild-Str. 2, D-85748 Garching by München, Germany

${ }^{9}$ Department of Physics and Astronomy, University of Leicester, University Road, Leicester LE1 7RH, UK
} 
of $R$ and $K_{s}$ magnitudes and redshifts. We detect the host galaxies for $80 \%$ of the GRBs in the sample, although only $42 \%$ have $K_{s}$-band detections, which confirms that GRB-selected host galaxies are generally blue. The sample is not uniformly blue, however, with two extremely red objects detected. Moreover, galaxies hosting GRBs with no optical/NIR afterglows, whose identification therefore relies on X-ray localizations, are significantly brighter and redder than those with an optical/NIR afterglow. This supports a scenario where GRBs occurring in more massive and dusty galaxies frequently suffer high optical obscuration. Our spectroscopic campaign has resulted in $77 \%$ now having redshift measurements, with a median redshift of $2.14 \pm 0.18$. TOUGH alone includes 17 detected $z>2$ Swift GRB host galaxies suitable for individual and statistical studies - a substantial increase over previous samples. Seven hosts have detections of the Ly $\alpha$ emission line and we can exclude an early indication that Ly $\alpha$ emission is ubiquitous among GRB hosts, but confirm that Ly $\alpha$ is stronger in GRB-selected galaxies than in flux-limited samples of Lyman break galaxies.

Subject headings: cosmology: observations - galaxies: fundamental parameters - galaxies: high-redshift - gamma rays: bursts - surveys

\section{Introduction}

Following the discovery of long-duration gamma-ray burst (GRB) optical afterglows (e.g., van Paradijs et al. 2000), evidence rapidly accumulated pointing to their origin in the deaths of massive stars (Galama et al. 1998; Bloom et al. 2002; Stanek et al. 2003; Hiorth et al. 2003). This soon led to their being touted as a potentially powerful means of discovering and reckoning star formation activity, which could bypass many of the biases that hamper other star formation measures (e.g., Wijers et al. 1998). The detection of GRBs is at once both independent of the luminosity of their host galaxy and, at high energies, unaffected by dust obscuration. They are also extremely luminous at all wavelengths, so that they can be used to perform transmission spectroscopy of their host galaxies, even where such hosts are too faint to be detected photometrically (e.g., Thöne et al. 2010). This also permits them to be found across the universe from very low to very high redshift: indeed

\footnotetext{
${ }^{1}$ Based on observations collected at the European Southern Observatory, Paranal, Chile (ESO Large Programme 177.A-0591). Reduced data and catalogs are made available at http://dark-cosmology.dk/TOUGH and http://archive.eso.org.
} 
GRB 090423 holds the record at $z=8.2$ for the most distant spectroscopically confirmed object known (Tanvir et al. 2009; Salvaterra et al. 2009).

The history of baryonic structure in the universe to a large extent revolves around the co-evolution of stars, galaxies, metals, and dust. In recent years, a range of methods has been applied to the identification and study of different populations of high-redshift galaxies, but all are variously affected, to a greater or lesser extent, by the problems of dust extinction, flux-limited samples, source confusion, and incomplete redshift determinations. Notable examples are: Lyman break galaxies (LBGs; Steidel et al. 2003), sub-mm galaxies (SMGs; e.g., Barger et al. 2000; Blain et al. 2002), Ly $\alpha$ emitters (LAEs; e.g., Fvnbo et al. 2003b, 2005; Sawicki \& Thompson 2006), distant red galaxies (DRGs; Daddi et al. 2004), damped Ly $\alpha$ absorbers (DLAs; Møller et al. 2002; Wolfe et al. 2005), and high-redshift dropout galaxies (e.g., Bouwens et al. 2010). Studies of these populations have led to major advances in our understanding of galaxy evolution, but our view remains far from complete, particularly at higher redshifts.

The major stumbling blocks to the routine use of GRBs as tools for the investigation of cosmic history have been the relatively slow buildup of statistically useful samples, and the disconnect between the high-energy properties of the burst and parameters useful for measuring the host and its star formation rate (SFR). These latter parameters, such as the redshift, the host-galaxy bolometric luminosity, metallicity, and dust content can only be determined from longer wavelength observations in the UV, optical, infrared, millimeter, and radio regimes.

Accurate X-ray localizations, as an intermediate step between the gamma-rays and the optical/infrared, have turned out to be critical to finding the afterglows and the host galaxies of GRBs. The first detections provided by BeppoSAX (Costa et al. 1997) heralded the afterglow era. Since 2005, Swift (Gehrels et al. 2004), with its autonomous slewing capability and onboard X-Ray Telescope (XRT, Burrows et al. 2005) has been extremely successful in this regard, delivering high-accuracy (few arcseconds) positions for approximately a hundred bursts every year. It has become the GRB facility par excellence thanks to its innovative, intelligent design, open access policy, energetic team, and active community support.

In spite of Swift's success, some of the problems that beset most other star-formation indicators also affect GRBs. Despite many attempts to infer distances from the prompt high-energy properties (e.g., Guidorzi 2005), UV/optical/NIR observations (and preferably spectroscopy) remain the only route to reliably find the redshift of a GRB or its host, and we must observe the hosts of GRBs directly across a range of wavelengths to connect SFR to GRB rate. Thus, to avoid optical biases in GRB selection requires a sample as complete as possible in redshift and host-galaxy properties. Swift's excellent X-ray localization 
completeness and large sample size provide a first opportunity for this to be attempted.

Several previous studies of GRB hosts have found that the majority of GRB hosts are faint, blue galaxies (Fruchter et al. 1999; Le Floc'h et al. 2003; Christensen et al. 2004) with low stellar masses (Castro Cerón et al. 2006, 2010; Savaglio et al. 2009). The GRBs generally occur within the UV-bright parts of their hosts (Bloom et al. 2002; Fruchter et al. 2006), consistent with their association with star formation. They are also typically fainter and have lower metallicities than the galaxies selected in Lyman-break surveys in the same redshift range (Jakobsson et al. 2005; Savaglio 2006; Fynbo et al. 2008b). Accounting for systematic incompleteness biases due to dust obscuration, i.e., the so-called dark burst problem (Fvnbo et al. 2001, 2009; De Pasquale et al. 2003; Jakobsson et al. 2004; Perlev et al. 2009; Krühler et al. 2011; Svensson et al. 2012; Rossi et al. 2012; Melandri et al. 2012), has also been attempted with some success (e.g., Jakobsson et al. 2006b; Fynbo et al. 2009).

Submillimeter (submm) and radio surveys of GRB hosts have shown that only a few could be ultraluminous infrared galaxies (Berger et al. 2003), indicating that obscured star formation is not dominant in GRB-selected galaxies (Tanvir et al. 2004). Moreover, the candidate submm bright GRB hosts are bluer, hotter, and at lower redshift than typical of SMGs (e.g., Berger et al. 2003; Michałowski et al. 2008).

Most of the studies mentioned above have been limited to fairly low-redshift samples $(z \lesssim 1.5)$, which calls for an extension of such statistical investigations to higher redshifts. Recently, more complete surveys (Greiner et al. 2011) have indicated the existence of a fraction of GRB host galaxies being more chemically evolved, more dusty and of higher mass (Krühler et al. 2011). This highlights the importance of minimizing optical selection effects in using GRBs as tracers of high-redshift star formation and the cosmic star-formation history (e.g., Tanvir et al. 2012; Salvaterra et al. 2012; Robertson \& Ellis 2012; Elliott et al. 2012; Basa et al. 2012; Trenti et al. 2012).

We have conducted a survey of galaxies hosting GRBs, suitable for statistical studies and for observations with other existing or future facilities. In particular, we have imaged 69 GRB host galaxies to deep limits in $R$ and $K_{s}$ and obtained follow-up spectroscopy to determine redshifts and study Ly $\alpha$ emission in the higher-redshift systems. The improved redshift completeness is also important for statistical studies of GRB properties themselves. The details of the survey design and target selection are described in Section 2. The observations are described in Section 3, along with the catalog of GRB and host galaxy properties, including magnitudes and redshifts. Section 4 provides a summary of some of the immediate scientific results and Section 5 concludes. More detailed discussion and results are presented in four companion papers by D. Malesani et al. (2012, in preparation), Jakobsson et al. (2012), Milvang-Jensen et al. (2012), and Krühler et al. (2012). Supplementary radio, Hub- 
ble Space Telescope (HST), and X-ray observations are reported in Michałowski et al. (2012), S. Schulze et al. (2012, in preparation), and D. Watson et al. (2012, in preparation).

All magnitudes given in this paper are in the Vega system. Throughout our work, we adopt a cosmology with $\Omega_{\mathrm{m}}=0.27, \Omega_{\Lambda}=0.73, H_{0}=71 \mathrm{~km} \mathrm{~s}^{-1} \mathrm{Mpc}^{-1}$.

\section{Target selection and sample definition}

A statistically meaningful and optically unbiased sample is essential in addressing issues such as the GRB redshift distribution, the importance of dust obscuration, and the determination of the burst and host luminosity functions (see also Salvaterra et al. 2012). We have applied the following selection criteria to provide a large and homogeneous sample of targets and so as to ensure that selection effects are minimized and well understood. The criteria are akin to those introduced by Jakobsson et al. (2006b) and refined by Fynbo et al. (2009).

1. Our starting point is the sample of Swift-detected GRBs. Swift is the only current facility which can provide a large, homogeneous, well-localized sample of GRBs. We pick only events triggered onboard by the Burst Alert Telescope (BAT) and disregard those (very few) discovered during ground analysis.

2. We limit ourselves to long-duration GRBs, and discard all events with $T_{90}<2 \mathrm{~s}$ (Kouveliotou et al. 1993), based on the catalog of Sakamoto et al. (2011).

3. The bursts must have a detected X-ray afterglow, the location of which is made available less than $12 \mathrm{hr}$ after the trigger. The requirement of the existence of an X-ray afterglow effectively makes our sample X-ray selected.

The above criteria are essentially constraints on the nature of the GRBs. The requirement on the timing in Criterion 3 additionally ensures that efficient afterglow searches could be performed. It also works as an effective Sun constraint, since those bursts with lines of sight close to the Sun upon discovery cannot be observed quickly. It should be noted that the fraction of long GRBs without a detected X-ray afterglow is very low, providing the XRT is repointed rapidly (see below).

Looking for the hosts of all these GRBs would be very demanding. We therefore apply further cuts, with the purpose of reducing the sample size in an unbiased way. Imposing restrictions 4-6 does not bias the sample toward optically bright afterglows; instead each GRB in the sample has favorable observing conditions, i.e., useful follow-up observations are likely to be secured. 
4. The Galactic foreground optical extinction as determined by Schlegel et al. (1998) must be $A_{V} \leqslant 0.5$ mag. This makes the detection level almost uniform (i.e., no bias against systems that happen to be faint just because of Galactic extinction). As a bonus, all fields have high Galactic latitude $\left(|b| \gtrsim 15^{\circ}\right)$ ensuring that crowding by foreground stars is not an issue (Figure 1).

5. As another visibility constraint, we require that the distance on the sky between the GRB and the Sun at the time of explosion is more than $55^{\circ}$ (similar to Jakobsson et al. $2006 \mathrm{~b})$. This is relevant since bursts closer to the Sun than this will offer very limited opportunity for ground follow-up. In practice, it is not a very demanding constraint because Criterion 3 effectively enforces a solar angle constraint of this order to satisfy the Swift Sun-avoidance condition for rapid slews for the narrow-field instruments (XRT and the Ultraviolet-Optical Telescope, UVOT) of $47^{\circ}$. In fact, during the survey period, only GRB 070810A had an X-ray afterglow announced within $12 \mathrm{hr}$ but had a discovery line of sight closer to the Sun.

6. We require that there is no nearby star or bright contaminating object that would render host-galaxy identification and spectroscopy difficult. To make this quantitative, we note that, under the typical observing conditions of our $R$-band imaging survey, bright stars affect a region of radius $r \approx 1.8+0.4 \times \exp [(20-R) / 2.05]$ arcsec, where $R$ is the USNO-B magnitude (Monet et al. 2003). This relation is valid in the range $11 \lesssim R \lesssim 19$. We thus require that no star with $R<19$ is located on the CCD detected within this distance from the X-ray error circle edge. In two cases, GRBs 060604 and 070721B, very bright stars $(R<10)$ at distances $\gtrsim 1^{\prime}$ would nominally violate the above limit, but their effect was minimized by offsetting the pointing such that they were located outside of the detector.

The aforementioned six criteria are applicable in principle to any survey of Swift GRB positions. Our survey was carried out with the VLT, and was restricted to bursts detected during a specific $2.5 \mathrm{yr}$ period, so that for this sample we applied two additional criteria.

7. Bursts triggered between 2005 March 1 and 2007 August 10. During this period Swift was fully operational and automatic slews were routinely enabled. There were 236 GRBs (229 triggered) in this time 2

\footnotetext{
${ }^{2}$ We do not consider GRBs 060204C, 060602B, and 070610, which were very likely due to Galactic sources (Sakamoto et al. 2006; Palmer et al. 2006; Kasliwal et al. 2008; Castro-Tirado et al. 2008).
} 
8. In order to be well placed for observation with the VLT we limit the declination range to be $-70^{\circ}<\delta<+27^{\circ}(\mathrm{J} 2000.0)$.

These criteria can easily be extended by using other observatories and different points in time.

After enforcing these criteria, we end up with a sample of 69 GRBs. The advantage of our selection is illustrated by the large success of afterglow follow-up for bursts in this subset. Based on Jochen Greiner's Web site 3 optical/NIR afterglows have been discovered for $75 \%$ (52/69) of the bursts in our sample, compared to $66 \%$ for the overall Swift sample. More importantly, spectroscopic redshifts are available for $55 \%$ of our targets (38 GRBs) compared to only $37 \%$ in the overall Swift sample.

Our final criterion concerns the localization accuracy of the GRBs.

9. The X-ray localization uncertainty must be better than or equal to 2 arcsec (90\% error radius). This constraint is dictated by the necessity to perform meaningful host searches. Larger error boxes would lead to too many ambiguous detections. For bursts without an optical/NIR localization, we adopt the best constrained position among the catalogs provided by Butler (2007b) and Evans et al. (2009). In principle, a more accurate localization could be available from optical or radio data, but we only base our selection on the X-ray position, to retain a dependence solely on high-energy properties.

We summarize the selection criteria in Table 1. Our final sample consists of 69 GRBs and is presented in Table 2. Figure 1 shows the distribution in the sky of all GRBs detected by Swift during the chosen time period with the TOUGH sample explicitly marked.

Below we discuss the effects of the various selections. Figure 2 shows the $1 \mathrm{~s}$ BAT $15-$ $150 \mathrm{keV}$ peak photon flux (from Sakamoto et al. 2011) versus the date. As can be seen, our targets uniformly sample the full BAT distribution and there is no obvious long-term change in the detection threshold as a function of time, which seems to be consistently at a level of about $0.4 \gamma \mathrm{cm}^{-2} \mathrm{~s}^{-1}$ (the faintest target GRB 060218 is detected at $0.25 \gamma \mathrm{cm}^{-2} \mathrm{~s}^{-1}$ ). This shows that we are starting from a stable, well-controlled set of events (Criterion 1), although we note that the effective trigger threshold does vary somewhat with many factors, such as the current background level, the position of the burst within the BAT field, and also the form of the particular GRB light curve (for example, the detection of some bursts

\footnotetext{
${ }^{3}$ http : //www.mpe.mpg.de/ jcg/grbgen.html
} 
is only made after integrating up more than the $1 \mathrm{~s}$ binning we consider here). Out of the 69 TOUGH bursts, 56 (13) were rate (image) triggers.

Our Criterion 3 is the detection of an X-ray afterglow. During the considered period, only 25 triggered GRBs were not detected (or observed) by XRT (11\% of the overall Swift sample). Restricting to long-duration bursts (Criterion 2) with prompt slew (less than $3600 \mathrm{~s})$, however, we find that only 5 events out of $180(2.8 \%)$ were not detected by XRT. This shows that, in practice, XRT selection has a negligible impact on the size of our sample, although it does mean that rare GRBs with very faint X-ray afterglows will be systematically underrepresented.

Figures 3 and 4 illustrate the distributions of the times to first XRT observation (taken from the Swift GRB tables; akin to but not identical to Criterion 3) and the Galactic $V$ band extinction (Schlegel et al. 1998, Criterion 4) for the TOUGH sample as well as the full distribution of GRBs.

Criterion 9 introduces a potential bias in our requirement of an accurate XRT position, which might lead us to favor optically bright afterglows. The accuracy of the X-ray position is influenced by many factors, several of which are not directly related to the GRB properties. For example, the astrometrically corrected positions (Butler 2007b) require the detection of serendipitous X-ray sources in the field other than the GRB. This depends more on the total exposure time than on the afterglow brightness. Figure 5 shows that the accuracy of X-ray positions has been stable during the considered time span. In fact, many of the UVOT-enhanced positions are dominated by the systematic error term which is independent of the afterglow brightness. There is a possible dearth of very accurate positions after 2007 March, which may be due to shallower exposures following a change of the Swift pointing strategy. In Figure 6, we investigate the impact of rejecting bursts without an accurate localization. We pick all GRBs which would pass selection criteria 1-8, regardless of the X-ray accuracy. We then plot their 90\% error radii (Butler 2007a; Evans et al. 2009) as a function of the count rate at $12 \mathrm{hr}$, obtained by interpolating or extrapolating the light curves posted online 5 (Evans et al. 2009). Figure 6 shows that, indeed, faint afterglows have on the average larger error radii, but the effect is very small (a factor of $\approx 3$ across more than two orders of magnitude in the count rate). Thus, among 71 GRBs considered here, only 2 are discarded (less than $3 \%$ ), showing that the impact on our sample is limited.

\footnotetext{
${ }^{4}$ http://swift.gsfc.nasa.gov/docs/swift/archive/grb_table/

${ }^{5}$ http://www.swift.ac.uk/xrt_curves/
} 


\section{Survey and catalogs}

Our observational program consists of deep $R$ and $K_{s}$ imaging and spectroscopy obtained with the FORS1, FORS2, ISAAC, and X-shooter instruments at the ESO VLT. All VLT observations were conducted at least 50 days after the GRB explosion in order to avoid possible contamination from the afterglow or an associated supernova. The possible afterglow contamination in our images is quantified in D. Malesani et al. (2012, in preparation). Here, we briefly describe the overall results, as summarized in Table 3. Detailed survey results are reported in four accompanying papers. Results on individual targets or preliminary results are reported in Ruiz-Velasco et al. (2007), Jakobsson et al. (2007, 2009, 2008, 2011a.b. c), Tanvir et al. (2008), Thöne et al. (2008, 2010), Jaunsen et al. (2008), Xu et al. (2009), Elíasdóttir et al. (2009), Ferrero et al. (2009), Fvnbo et al. (2009, 2008a),

Malesani et al. (2009, 2010), Svensson et al. (2012), and Schulze et al. (2012). In Tables 2 and 3, we also list 10 extra hosts that were observed for our program, but that do not obey all of the TOUGH sample criteria.

\subsection{Optical imaging}

For the purposes of identification of hosts and brightness estimation we have obtained deep images of the targets in the $R$ band (typically rest-frame UV) to magnitude limits in the range $R=26-28 \mathrm{mag}$. The seeing FWHM of the $R$-band images ranges from 0.50 to $1^{\prime \prime} .26$ with a median of 0 ' 71 . D. Malesani et al. (2012, in preparation) discuss our procedure for identifying a host galaxy, including a quantification of the chance probability of wrongly identifying an unrelated galaxy in the error circle as the host. We note that there is always a small risk of wrong host identification in both imaging and spectroscopy. While this could lead to erroneous conclusions when discussing individual systems, it will have little effect on statistical results inferred from a sufficiently large sample, like the one presented here, as long as the probability for false identification is low.

We have identified host galaxies for 55 systems, corresponding to $80 \%$ of the sample. The coordinates of the identified host galaxies are reported in Table 3. In Figure 7 , we show a mosaic of GRB host galaxies that are only localized by the XRT (a mosaic of all identified host galaxies is presented in D. Malesani et al. (2012, in preparation). These 17 galaxies (25\% of our sample) are typically missed from other surveys. The observed magnitudes are shown in Figure 8, It is evident that host galaxies are generally sub-luminous, in the sense of being fainter than $L^{*}$ at $z=0$. We note that the simple detection of a host galaxy in the $R$ band can be used to set an upper limit to the redshift of the GRB of $z \lesssim 5.6$ (Ruiz-Velasco et al. 2007; Jakobsson et al. 2012). 


\section{2. $\quad$ Near-infrared imaging}

To obtain basic color information for the targets we also obtained $K_{s}$-band imaging (typically rest-frame visual) to a limiting magnitude of about 22. The seeing FWHM of the $K_{s}$-band images ranges from $00^{\prime} 34$ to $00^{\prime \prime} 83$ with a median of 0 ' 51 . These data allow detection of extremely red objects (EROs, $R-K_{s}>5$ ) for all $R$-band-detected targets. Although the majority of previously studied GRB hosts have had blue optical-IR colors, a small number of ERO hosts have been identified (e.g., Levan et al. 2006a; Berger et al. 2007; Hunt et al. 2011; Svensson et al. 2012; Rossi et al. 2012). The resulting $R-K_{s}$ colors for our sample are shown in Figure 8. These are roughly consistent with Le Floc'h et al. (2003). All host galaxies detected in $K_{s}$ were also detected in $R$. We have identified two EROs among our sample, the host galaxies of GRB 050714B and GRB 070808 which both have $R-K_{s} \approx 5$.

\subsection{Spectroscopy}

The identification of the hosts was used to determine their suitability for spectroscopic follow-up. For targets with no afterglow absorption redshift that are sufficiently bright for spectroscopic redshift determination (host galaxies generally are emission-line galaxies) we have obtained spectra with the purpose of making the redshift determinations for our sample as complete as possible. This is a crucial step in making any statistical inferences about the redshift distribution of GRBs and their host galaxies, in particular with reference to the star formation history of the universe. However, we note that the selection of galaxies at this stage based on their apparent $R$-band magnitudes could introduce an optical bias into our sample, and that securing redshifts for the remaining (fainter) galaxies remains a priority. As

reported by Jakobsson et al. (2012) and Krühler et al. (2012), we have obtained spectra of 23 host galaxies in the TOUGH sample for which we have determined 15 new redshifts (and, unfortunately, found that 3 previously reported redshifts of the sample were erroneous). In total, we now have redshifts for 53 galaxies out of 69 in the sample. Moreover, even in cases where spectroscopy was unsuccessful in determining a redshift, spectra and imaging were used to set useful constraints, e.g., from an upper limit of the position of the Lyman limit or Ly $\alpha$ breaks or from the absence of emission lines.

\section{Discussion}

We have verified that there are no obvious trends in the redshifts or magnitudes of the TOUGH host galaxies as a function of GRB epoch. 
Below we summarize some of the results of the TOUGH survey, with special emphasis on the implications of having compiled an X-ray selected sample of GRB host galaxies, and obtaining photometry and spectroscopy of a sample of GRB host galaxies extending to faint and high-redshift systems.

\subsection{Redshift distribution}

In Figure 9, we show the redshift distribution prior to our survey and compare to the redshift distribution resulting from our survey. All redshifts are given in Table 3. The new redshift distribution is more smooth with a conspicuous peak at around redshift 2 with a median redshift of $2.14 \pm 0.18$. The median redshift of the entire TOUGH sample (i.e., including systems with no redshift constraint) is robustly determined to be between 1.5 and 2.5 (Table 4). As discussed by Jakobsson et al. (2012) our redshifts and redshift constraints allow us to distinguish models for the GRB rate and luminosity function and formation biases.

To illustrate the importance of sample selection criteria for the determination of the median redshift for GRBs we plot the redshift completeness as a function of BAT (15$150 \mathrm{keV}$ ) peak photon flux in Figure 10. The median $1 \mathrm{~s}$ peak flux for the TOUGH sample is

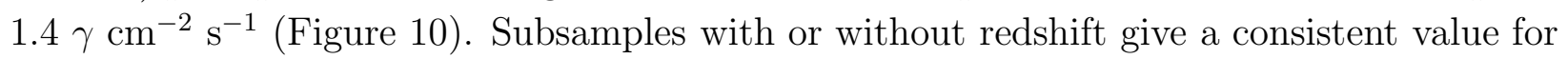
the median $1 \mathrm{~s}$ peak flux, as do subsamples with or without an optical/NIR afterglow. About $75 \%$ of the GRBs in our sample have lower peak photon flux than the cutoff adopted by Salvaterra et al. (2012) ( $\left.\geq 2.6 \gamma \mathrm{cm}^{-2} \mathrm{~s}^{-1}\right)$. Our redshift completeness is in the range $70 \%-$ $80 \%$ below that value and reaches almost $90 \%$ slightly above. The median redshift increases almost monotonically with lower peak flux, from 1.38 at a cutoff at $2.6 \gamma \mathrm{cm}^{-2} \mathrm{~s}^{-1}$ to 2.12

for a cutoff at $0.4 \gamma \mathrm{cm}^{-2} \mathrm{~s}^{-1}$. Tying to the BATSE luminosity function, Salvaterra et al. (2012) modeled their results and predicted a median redshift of $z=2.05 \pm 0.15$ to that limit, consistent with our value.

However, obtaining redshifts of fainter galaxies may shift the median redshift somewhat higher. As indicated by Krühler et al. (2012) the faint host galaxies targeted with X-shooter have been found at significantly higher redshifts compared to brighter targets. We therefore suspect that the remaining very faint host galaxies may lead to even higher average redshifts. Figure 11] shows the median redshift as a function of the median $R$-band magnitude of the hosts (the corresponding values are given in Table 4). The median redshift of the TOUGH sub-samples is lowest for the bright hosts for which the redshift is determined from emission lines using FORS, higher for fainter hosts where the redshift is determined using X-shooter, and higher still for bursts where the host remains undetected. $K_{s}$-detected host galaxies are 
at significantly lower redshifts than galaxies which are not detected in $K_{s}$. The overall trend seems to be consistent with the expectation that fainter GRB host galaxies are on average at higher redshifts.

TOUGH for the first time picks up high-redshift GRB hosts in significant numbers. The compilations of Chen et al. (2009) and Savaglio et al. (2009) count eight optically detected GRB host galaxies at $z>2$ of which two are Swift GRBs. Table 3 lists a total of 18 TOUGHdetected GRB host galaxies at $z>2$, of which 17 are new, as well as one non-TOUGH $z>2$ GRB host galaxy. Such a large sample opens the field for more detailed studies of individual systems and of high-redshift GRB hosts as a whole (see also Section 4.3).

\subsection{Galaxy colors and magnitudes}

Of special interest is the study of dark bursts which are believed to be caused mostly by dust obscuration (e.g., Diorgovski et al. 2001; Rol et al. 2007; Jaunsen et al. 2008; Tanvir et al. 2008; Perley et al. 2009; Krühler et al. 2011; Svensson et al. 2012; Rossi et al. 2012). Hence, it is expected that the properties of the host galaxies of XRT-only GRBs may be different from those hosting GRBs with detected optical/NIR afterglows.

Figure 12 highlights the difference between systems with and without an optical/NIR afterglow. There is a significant dearth of XRT-only galaxies at very faint magnitudes in the $R$ band. This is also reflected in the median $R$-band magnitude of $25.8 \pm 0.3$ for galaxies hosting an optically/NIR-detected afterglow, as opposed to $24.4 \pm 0.4$ for those with undetected ones (Table 4 ). XRT-only localised host galaxies are also relatively brighter in $K_{s}$ (median magnitude of $K_{s}=21.1 \pm 0.3$ opposed to $K_{s}>22.5$ for galaxies hosting GRBs with optical/NIR afterglows). A clue to the origin of these remarkable differences comes from the lower panel of Figure 12 which demonstrates that XRT-only hosts have equivalent X-ray absorption column densities at $z=0$ extending to much higher values (see also Krühler et al. 2012). These differences clearly demonstrate that statistical studies based on optically selected GRBs will be strongly biased.

We note that our procedure of identifying the host as the brightest galaxy inside the XRT error circle naturally will lead to a bias toward brighter galaxies if any of the identifications are incorrect. To quantify this bias we tested our host identification procedure in the XRT error circles of GRBs with an optical/NIR afterglow. We found that in 6 out of 50 cases $(12 \%)$ we would misidentify the host (this would indicate that about $1-2$ of the galaxies in Figure 7 may have been misidentified). This procedure led to a biased median $R$-band magnitude of $25.5 \pm 0.2$ for the XRT error circles of GRB with optical/NIR afterglows but 
XRT-only identified host galaxies. Hence, the difference between host galaxies with and without optical/NIR afterglows remains significant.

Regarding systems with both $R$ and $K_{s}$ detections, we find that the median magnitude of hosts of GRBs with or without optical/NIR afterglows is consistently $R \approx 24.3 \pm 0.3$. However, the median colors of XRT-only GRB host galaxies detected in the $K_{s}$ band are significantly redder, $R-K_{s}=3.7 \pm 0.3$, than those for which an optical/NIR afterglow was detected, $R-K_{s}=2.8 \pm 0.1$. While this difference in color could partly be a redshift effect, their larger X-ray absorption column densities suggest that GRBs without an optical/NIR afterglow suffer stronger attenuation due to dust and that dust extinction causes optical/NIR GRB afterglows to drop out of detection. This is in line with other studies which find that host galaxies of dark GRBs are redder and brighter (more massive) than those of GRBs with optical afterglows (Krühler et al. 2011; Svensson et al. 2012). A more detailed discussion of the $R-K_{s}$ distribution as a function of redshift and a comparison to other high-redshift galaxies are presented in D. Malesani (2012, in preparation).

\subsection{Star formation in GRB host galaxies}

Motivated by the fact that, before our survey, five out of a possible five hosts in a pre-Swift GRB sample were found to be LAEs (Fynbo et al. 2002, 2003a; Jakobsson et al. 2005), we have also performed spectroscopic observations of systems with known redshift in the redshift range 1.8-4.5 where the Ly $\alpha$ emission line can be best observed. Remarkably, as reported by Milvang-Jensen et al. (2012), only 7 out of 20 galaxies targeted have secure detection of $\operatorname{Ly} \alpha$ emission while upper limits on the $\operatorname{Ly} \alpha$ equivalent width are derived for another 7 galaxies from detecting the continuum in the spectra (Figure 8). The origin of the lower (but more representative) success rate is likely a combination of lower intrinsic luminosities of the galaxies (and hence lower average Ly $\alpha$ luminosities) and lower equivalent widths, at least partly due to higher intrinsic extinction in the host galaxy. The Ly $\alpha$ emission is still stronger than in flux-limited samples of LBGs. Moreover, by comparison to the afterglow redshifts, we find that the velocity centroid of the Ly $\alpha$ line is redshifted by 200$600 \mathrm{~km} \mathrm{~s}^{-1}$ with respect to the systemic velocity, indicative of outflows in the host galaxies (e.g., Adelberger et al. 2003; Verhamme et al. 2006; Laursen et al. 2009).

In order to assess the SFRs of GRB hosts, we have performed radio observations of TOUGH GRB hosts at $z<1$ (Michałowski et al. 2012). This redshift limit was chosen to obtain meaningful limits on SFRs. We did not detect any TOUGH GRB hosts, which indicates that their average SFR is below $\sim 15 M_{\odot} \mathrm{yr}^{-1}$. We also found that at least $65 \%$ of GRB hosts at $z<1$ have $\mathrm{SFR}<100 M_{\odot} \mathrm{yr}^{-1}$ and that at least $92 \%$ of them have $A_{V}<3$ 
mag. The obtained limits allowed us to conclude that the distribution of SFRs and dust attenuation of GRB hosts at $z<1$ is consistent with that of other star-forming galaxies at similar redshifts.

Finally, in S. Schulze et al. (2012, in preparation) the magnitudes are used to determine the luminosity function of GRB-selected galaxies. In particular, determining the slope of the faint end power-law part of the luminosity function is important in determining whether such galaxies constitute a major fraction of the star formation in the universe (Fvnbo et al. 2001, 2002; Jakobsson et al. 2005, 2012; Robertson \& Ellis 2012; Tanvir et al. 2012).

\section{Summary}

Long-duration GRBs provide a complementary view of the nature of "typical" starforming galaxies over most of cosmic history. However, to date, most samples of GRB hosts have been heterogeneous and had a strong bias toward GRBs with detected optical afterglows. In this paper we have described a large, homogeneous sample of hosts targeted with the VLT, using X-ray positions where no optical/NIR afterglow was found. We report the main catalog in Table 3 . We detect the majority of the hosts, namely $80 \%$, in the $R$-band down to a detection limit of $\sim 27.5$, including 17 systems at $z>2$. Within the TOUGH sample, we detect 54 hosts, of which 32 were not previously detected. GRB host galaxies cover a wide range of luminosities from $<0.1 L^{*}$ to $L^{*}$. From the $K_{s}$-band imaging we confirm the result of more biased studies that GRB host galaxies tend to be blue. We also find that galaxies with no optical/NIR afterglow are significantly brighter and redder than those with an optical/NIR afterglow, highlighting the importance of TOUGH in being X-ray selected. With our newly reported redshifts, the redshift distribution consisting of $77 \%$ of the GRBs, now appears smooth with a median redshift above 2 .

Our sample will form the basis for statistical studies of GRB host galaxies, such as their luminosity function, colors and SFRs, redshift distribution, Ly $\alpha$ properties, radio emission, and X-ray absorption properties.

Future observational work will focus on completing the sample, in particular in redshift, and to complement it at other wavelengths, in particular, radio, sub-mm (ALMA, Herschel), and NIR/MIR (Spitzer, HST, JWST). It is also useful for selecting subsamples for further study, such as SED studies or emission-line diagnostics, e.g., metallicity determination. Finally, it is straightforward to enlarge in a well-defined way, in time or declination, as we are doing with VLT/X-shooter and Gemini. 
We thank the members of the former GRACE collaboration as well as participants of the former EU FP5 Research Training Network "Gamma-Ray Bursts: An Enigma and a Tool", for their initial support of and continued interest in this project. We are particularly grateful to Paul M. Vreeswijk for his enthusiastic contributions to the project. We are grateful to Scott Barthelmy, Nat Butler, Phil Evans, Samantha Oates, and Patricia Schady for their assistance in defining our sample selection criteria. We acknowledge the use of Jochen Greiner's GRB Web site. This work made use of data supplied by the UK Swift Science Data Centre at the University of Leicester. D.M. acknowledges support from the Instrument Center for Danish Astrophysics. P.J. and S.S. acknowledge support by a Project Grant from the Icelandic Research Fund. J.P.U.F. and B.M.-J. acknowledge support from the ERC-StG grant EGGS-278202. The research activities of J.G. are supported by the Spanish research programs AYA2008-03467/ESP and AYA2009-14000-C03-01. M.J.M. acknowledges the support of the Science and Technology Facilities Council. The Dark Cosmology Centre is funded by the Danish National Research Foundation.

Facilities: VLT:Antu(FORS2,ISAAC), VLT:Kueyen (FORS1,XSHOOTER), Swift(BAT,XRT) 


\section{REFERENCES}

Adelberger, K. L., Steidel, C. C., Shapley, A. E., \& Pettini, M. 2003, ApJ, 584, 45

Afonso, P., Greiner, J., Pian, E., Covino, S., Malesani, D., Küpcü Yoldaş, A., Krühler, T., Clemens, C., McBreen, S., Rau, A., Giannios, D., \& Hjorth, J. 2011, A\&A, 526, A154

Angelini, L., Barthelmy, S., Burrows, D., Chester, M., Cummings, J., Gehrels, N., Goad, M., Krimm, H., Marshall, F., \& Palmer, D. 2006, GRB Coordinates Network, 4848, 1

Asfandyarov, I., Pozanenko, A., \& Ibrahimov, M. 2006, GRB Coordinates Network, 5434, 1

Band, D. L., Barthelmy, S., Boyd, P., Brown, P., Burrows, D., Chester, M., Cummings, J., Gehrels, N., Markwardt, C., Nousek, J., Page, K., Pagani, C., Palmer, D., Sakamoto, T., \& Racusin, J. 2005, GRB Coordinates Network, 3748, 1

Barger, A. J., Cowie, L. L., \& Richards, E. A. 2000, AJ, 119, 2092

Basa, S., Cuby, J. G., Savaglio, S., Boissier, S., Clément, B., Flores, H., Le Borgne, D., \& Mazure, A. 2012, A\&A, 542, A103

Beardmore, A., Page, K., Goad, M., \& Kennea, J. 2005, GRB Coordinates Network, 4282, 1

Berger, E., Cowie, L. L., Kulkarni, S. R., Frail, D. A., Aussel, H., \& Barger, A. J. 2003, ApJ, 588,99

Berger, E., Fox, D. B., Kulkarni, S. R., Frail, D. A., \& Djorgovski, S. G. 2007, ApJ, 660, 504

Berger, E., \& Gladders, M. 2006, GRB Coordinates Network, 5170, 1

Berger, E., Kulkarni, S. R., Fox, D. B., Soderberg, A. M., Harrison, F. A., Nakar, E., Kelson, D. D., Gladders, M. D., Mulchaey, J. S., Oemler, A., Dressler, A., Cenko, S. B., Price, P. A., Schmidt, B. P., Frail, D. A., Morrell, N., Gonzalez, S., Krzeminski, W., Sari, R., Gal-Yam, A., Moon, D.-S., Penprase, B. E., Jayawardhana, R., Scholz, A., Rich, J., Peterson, B. A., Anderson, G., McNaught, R., Minezaki, T., Yoshii, Y., Cowie, L. L., \& Pimbblet, K. 2005, ApJ, 634, 501

Blain, A. W., Smail, I., Ivison, R. J., Kneib, J.-P., \& Frayer, D. T. 2002, Phys. Rep., 369, 111

Bloom, J. S., \& Alatalo, K. 2005, GRB Coordinates Network, 3984, 1

Bloom, J. S., Kulkarni, S. R., \& Djorgovski, S. G. 2002, AJ, 123, 1111 
Blustin, A., Barthelmy, S., Burrows, D., Chester, M., Cummings, J., Gehrels, N., Kennea, J., Marshall, F., Palmer, D., \& Sakamoto, T. 2005a, GRB Coordinates Network, 3849, 1

Blustin, A. J., Band, D., Barthelmy, S., Boyd, P., Capalbi, M., Holland, S. T., Marshall, F. E., Mason, K. O., Perri, M., Poole, T., Roming, P., Rosen, S., Schady, P., Still, M., Zhang, B., Angelini, L., Barbier, L., Beardmore, A., Breeveld, A., Burrows, D. N., Cummings, J. R., Cannizzo, J., Campana, S., Chester, M. M., Chincarini, G., Cominsky, L. R., Cucchiara, A., de Pasquale, M., Fenimore, E. E., Gehrels, N., Giommi, P., Goad, M., Gronwall, C., Grupe, D., Hill, J. E., Hinshaw, D., Hunsberger, S., Hurley, K. C., Ivanushkina, M., Kennea, J. A., Krimm, H. A., Kumar, P., Landsman, W., La Parola, V., Markwardt, C. B., McGowan, K., Mészáros, P., Mineo, T., Moretti, A., Morgan, A., Nousek, J., O'Brien, P. T., Osborne, J. P., Page, K., Page, M. J., Palmer, D. M., Parsons, A. M., Rhoads, J., Romano, P., Sakamoto, T., Sato, G., Tagliaferri, G., Tueller, J., Wells, A. A., \& White, N. E. 2006, ApJ, 637, 901

Blustin, A. J., \& Page, M. J. 2006, GRB Coordinates Network, 5219, 1

Blustin, A. J., Parsons, A., Holland, S. T., Meszaros, P., Chester, M., \& Gehrels, N. 2005b, GRB Coordinates Network, 4107, 1

Bouwens, R. J., Illingworth, G. D., Oesch, P. A., Stiavelli, M., van Dokkum, P., Trenti, M., Magee, D., Labbé, I., Franx, M., Carollo, C. M., \& Gonzalez, V. 2010, ApJ, 709, L133

Burrows, D. N., Hill, J. E., Nousek, J. A., Kennea, J. A., Wells, A., Osborne, J. P., Abbey, A. F., Beardmore, A., Mukerjee, K., Short, A. D. T., Chincarini, G., Campana, S., Citterio, O., Moretti, A., Pagani, C., Tagliaferri, G., Giommi, P., Capalbi, M., Tamburelli, F., Angelini, L., Cusumano, G., Bräuninger, H. W., Burkert, W., \& Hartner, G. D. 2005, Space Sci. Rev., 120, 165

Butler, N. R. 2007a, ApJ, 656, 1001

-. 2007b, AJ, 133, 1027

Campana, S., Mangano, V., Blustin, A. J., Brown, P., Burrows, D. N., Chincarini, G., Cummings, J. R., Cusumano, G., Della Valle, M., Malesani, D., Mészáros, P., Nousek, J. A., Page, M., Sakamoto, T., Waxman, E., Zhang, B., Dai, Z. G., Gehrels, N., Immler, S., Marshall, F. E., Mason, K. O., Moretti, A., O’Brien, P. T., Osborne, J. P., Page, K. L., Romano, P., Roming, P. W. A., Tagliaferri, G., Cominsky, L. R., Giommi, P., Godet, O., Kennea, J. A., Krimm, H., Angelini, L., Barthelmy, S. D., Boyd, P. T., Palmer, D. M., Wells, A. A., \& White, N. E. 2006, Nature, 442, 1008 
Castro Cerón, J. M., Michałowski, M. J., Hjorth, J., Malesani, D., Gorosabel, J., Watson, D., Fynbo, J. P. U., \& Morales Calderón, M. 2010, ApJ, 721, 1919

Castro Cerón, J. M., Michałowski, M. J., Hjorth, J., Watson, D., Fynbo, J. P. U., \& Gorosabel, J. 2006, ApJ, 653, L85

Castro-Tirado, A. J., de Ugarte Postigo, A., Gorosabel, J., Jelínek, M., Fatkhullin, T. A., Sokolov, V. V., Ferrero, P., Kann, D. A., Klose, S., Sluse, D., Bremer, M., Winters, J. M., Nuernberger, D., Pérez-Ramírez, D., Guerrero, M. A., French, J., Melady, G., Hanlon, L., McBreen, B., Leventis, K., Markoff, S. B., Leon, S., Kraus, A., Aceituno, F. J., Cunniffe, R., Kubánek, P., Vítek, S., Schulze, S., Wilson, A. C., Hudec, R., Durant, M., González-Pérez, J. M., Shahbaz, T., Guziy, S., Pandey, S. B., Pavlenko, L., Sonbas, E., Trushkin, S. A., Bursov, N. N., Nizhelskij, N. A., Sánchez-Fernández, C., \& Sabau-Graziati, L. 2008, Nature, 455, 506

Chary, R., Berger, E., \& Cowie, L. 2007, ApJ, 671, 272

Chen, H.-W., Perley, D. A., Pollack, L. K., Prochaska, J. X., Bloom, J. S., DessaugesZavadsky, M., Pettini, M., Lopez, S., Dall'aglio, A., \& Becker, G. D. 2009, ApJ, 691, 152

Chen, H.-W., Prochaska, J. X., Bloom, J. S., \& Thompson, I. B. 2005, ApJ, 634, L25

Chen, H.-W., Prochaska, J. X., Herbert-Fort, S., Christlein, D., \& Cortes, S. 2007, GRB Coordinates Network, 6217, 1

Chen, Y. C., Lee, Y. H., Huang, K. Y., Ip, W. H., \& Urata, Y. 2006, GRB Coordinates Network, 5797, 1

Chester, M. M., Marshall, F. E., \& Cummings, J. R. 2007, GRB Coordinates Network, 6735, 1

Chester, M. M., Wang, X. Y., Cummings, J. R., Grupe, D., Hunsberger, S. D., La Parola, V., Marshall, F. E., Mineo, T., Oates, S. R., Page, M. J., \& Roming, P. 2008, in AIP Conf. Proc. 1000, Gamma-Ray Bursts 2007, ed. M. Galassi, D. Palmer, \& E. Fenimore (Melville, NY: AIP), 421

Christensen, L., Hjorth, J., \& Gorosabel, J. 2004, A\&A, 425, 913

Costa, E., Frontera, F., Heise, J., Feroci, M., in’t Zand, J., Fiore, F., Cinti, M. N., Dal Fiume, D., Nicastro, L., Orlandini, M., Palazzi, E., Rapisarda\#, M., Zavattini, G., Jager, R., Parmar, A., Owens, A., Molendi, S., Cusumano, G., Maccarone, M. C., 
Giarrusso, S., Coletta, A., Antonelli, L. A., Giommi, P., Muller, J. M., Piro, L., \& Butler, R. C. 1997, Nature, 387, 783

Covino, S., Malesani, D., \& Tagliaferri, G. 2006, GRB Coordinates Network, 5604, 1

Cummings, J., Mangano, V., Angelini, L., Barbier, L., Barthelmy, S., Burrows, D. N., Chester, M., Fenimore, E., Gehrels, N., Hullinger, D., Kennea, J., Krimm, H., La Parola, V., Levan, A., Markwardt, C., Mineo, T., Norris, J., O’Brien, P., Palmer, D., Parsons, A., Rol, E., Romano, P., Sakamoto, T., Sato, G., \& Tueller, J. 2006, GRB Coordinates Network, 4608, 1

Cummings, J. R., Barthelmy, S. D., Evans, P. A., Gehrels, N., Holland, S. T., Kennea, J. A., Krimm, H. A., Mangano, V., Marshall, F. E., Page, K. L., Palmer, D. M., Romano, P., Sato, G., Stamatikos, M., Starling, R. L. C., Troja, E., Ukwatta, T. N., \& vanden Berk, D. E. 2007, GRB Coordinates Network, 6718, 1

Daddi, E., Cimatti, A., Renzini, A., Fontana, A., Mignoli, M., Pozzetti, L., Tozzi, P., \& Zamorani, G. 2004, ApJ, 617, 746

Dai, X., Halpern, J. P., Morgan, N. D., Armstrong, E., Mirabal, N., Haislip, J. B., Reichart, D. E., \& Stanek, K. Z. 2007, ApJ, 658, 509

Dall'Aglio, A., Wisotzki, L., \& Worseck, G. 2008, A\&A, 491, 465

D’Avanzo, P., Piranomonte, S., Magazzu, A., \& Mainella, G. 2006, GRB Coordinates Network, 5151, 1

de Pasquale, M., Goad, M., Blustin, A. J., Chester, M., Angelini, L., \& Gehrels, N. 2005, GRB Coordinates Network, 3960, 1

de Pasquale, M., Oates, S. R., Page, M. J., Burrows, D. N., Blustin, A. J., Zane, S., Mason, K. O., Roming, P. W. A., Palmer, D., Gehrels, N., \& Zhang, B. 2007, MNRAS, 377, 1638

De Pasquale, M., Piro, L., Perna, R., Costa, E., Feroci, M., Gandolfi, G., in 't Zand, J., Nicastro, L., Frontera, F., Antonelli, L. A., Fiore, F., \& Stratta, G. 2003, ApJ, 592, 1018

de Pasquale, M., \& Ziaeepour, H. 2007, GRB Coordinates Network, 6650, 1

de Ugarte Postigo, A., Jelinek, M., Tristram, P., Bond, I., Yock, P., Hearnshaw, J., \& Castro-Tirado, A. J. 2007, GRB Coordinates Network, 6321, 1 
D’Elia, V., Fiore, F., Meurs, E. J. A., Chincarini, G., Melandri, A., Norci, L., Pellizza, L., Perna, R., Piranomonte, S., Sbordone, L., Stella, L., Tagliaferri, G., Vergani, S. D., Ward, P., Angelini, L., Antonelli, L. A., Burrows, D. N., Campana, S., Capalbi, M., Cimatti, A., Costa, E., Cusumano, G., Della Valle, M., Filliatre, P., Fontana, A., Frontera, F., Fugazza, D., Gehrels, N., Giannini, T., Giommi, P., Goldoni, P., Guetta, D., Israel, G., Lazzati, D., Malesani, D., Marconi, G., Mason, K., Mereghetti, S., Mirabel, F., Molinari, E., Moretti, A., Nousek, J., Perri, M., Piro, L., Stratta, G., Testa, V., \& Vietri, M. 2007, A\&A, 467, 629

Della Valle, M., Chincarini, G., Panagia, N., Tagliaferri, G., Malesani, D., Testa, V., Fugazza, D., Campana, S., Covino, S., Mangano, V., Antonelli, L. A., D’Avanzo, P., Hurley, K., Mirabel, I. F., Pellizza, L. J., Piranomonte, S., \& Stella, L. 2006a, Nature, 444, 1050

Della Valle, M., Malesani, D., Bloom, J. S., Benetti, S., Chincarini, G., D’Avanzo, P., Foley, R. J., Covino, S., Melandri, A., Piranomonte, S., Tagliaferri, G., Stella, L., Gilmozzi, R., Antonelli, L. A., Campana, S., Chen, H.-W., Filliatre, P., Fiore, F., Fugazza, D., Gehrels, N., Hurley, K., Mirabel, I. F., Pellizza, L. J., Piro, L., \& Prochaska, J. X. 2006b, ApJ, 642, L103

Djorgovski, S. G., Frail, D. A., Kulkarni, S. R., Bloom, J. S., Odewahn, S. C., \& Diercks, A. 2001, ApJ, 562, 654

Elíasdóttir, Á., Fynbo, J. P. U., Hjorth, J., Ledoux, C., Watson, D. J., Andersen, A. C., Malesani, D., Vreeswijk, P. M., Prochaska, J. X., Sollerman, J., \& Jaunsen, A. O. 2009, ApJ, 697, 1725

Elliott, J., Greiner, J., Khochfar, S., Schady, P., Johnson, J. L., \& Rau, A. 2012, A\&A, 539, A113

Evans, P. A., Beardmore, A. P., Page, K. L., Osborne, J. P., O’Brien, P. T., Willingale, R., Starling, R. L. C., Burrows, D. N., Godet, O., Vetere, L., Racusin, J., Goad, M. R., Wiersema, K., Angelini, L., Capalbi, M., Chincarini, G., Gehrels, N., Kennea, J. A., Margutti, R., Morris, D. C., Mountford, C. J., Pagani, C., Perri, M., Romano, P., \& Tanvir, N. 2009, MNRAS, 397, 1177

Ferrero, P., Klose, S., Kann, D. A., Savaglio, S., Schulze, S., Palazzi, E., Maiorano, E., Böhm, P., Grupe, D., Oates, S. R., Sánchez, S. F., Amati, L., Greiner, J., Hjorth, J., Malesani, D., Barthelmy, S. D., Gorosabel, J., Masetti, N., \& Roth, M. M. 2009, A\&A, 497, 729 
Fox, A. J., Ledoux, C., Vreeswijk, P. M., Smette, A., \& Jaunsen, A. O. 2008, A\&A, 491, 189

Fruchter, A. S., Levan, A. J., Strolger, L., Vreeswijk, P. M., Thorsett, S. E., Bersier, D., Burud, I., Castro Cerón, J. M., Castro-Tirado, A. J., Conselice, C., Dahlen, T., Ferguson, H. C., Fynbo, J. P. U., Garnavich, P. M., Gibbons, R. A., Gorosabel, J., Gull, T. R., Hjorth, J., Holland, S. T., Kouveliotou, C., Levay, Z., Livio, M., Metzger, M. R., Nugent, P. E., Petro, L., Pian, E., Rhoads, J. E., Riess, A. G., Sahu, K. C., Smette, A., Tanvir, N. R., Wijers, R. A. M. J., \& Woosley, S. E. 2006, Nature, 441, 463

Fruchter, A. S., Thorsett, S. E., Metzger, M. R., Sahu, K. C., Petro, L., Livio, M., Ferguson, H., Pian, E., Hogg, D. W., Galama, T., Gull, T. R., Kouveliotou, C., Macchetto, D., van Paradijs, J., Pedersen, H., \& Smette, A. 1999, ApJ, 519, L13

Fynbo, J. P. U., Hjorth, J., Malesani, D., Sollerman, J., Watson, D., Jakobsson, P., Gorosabel, J., \& Jaunsen, A. O. 2008a, in The Eleventh Marcel Grossmann Meeting On Recent Developments in Theoretical and Experimental General Relativity, Gravitation and Relativistic Field Theories, ed. H. Kleinert, R. T. Jantzen, \& R. Ruffini (Singapore: World Scientific), 726

Fynbo, J. P. U., Jakobsson, P., Möller, P., Hjorth, J., Thomsen, B., Andersen, M. I., Fruchter, A. S., Gorosabel, J., Holland, S. T., Ledoux, C., Pedersen, H., Rhoads, J., Weidinger, M., \& Wijers, R. A. M. J. 2003a, A\&A, 406, L63

Fynbo, J. P. U., Jakobsson, P., Prochaska, J. X., Malesani, D., Ledoux, C., de Ugarte Postigo, A., Nardini, M., Vreeswijk, P. M., Wiersema, K., Hjorth, J., Sollerman, J., Chen, H.-W., Thöne, C. C., Björnsson, G., Bloom, J. S., Castro-Tirado, A. J., Christensen, L., De Cia, A., Fruchter, A. S., Gorosabel, J., Graham, J. F., Jaunsen, A. O., Jensen, B. L., Kann, D. A., Kouveliotou, C., Levan, A. J., Maund, J., Masetti, N., Milvang-Jensen, B., Palazzi, E., Perley, D. A., Pian, E., Rol, E., Schady, P., Starling, R. L. C., Tanvir, N. R., Watson, D. J., Xu, D., Augusteijn, T., Grundahl, F., Telting, J., \& Quirion, P.-O. 2009, ApJS, 185, 526

Fynbo, J. P. U., Krog, B., Nilsson, K., Björnsson, G., Hjorth, J., Jakobsson, P., Ledoux, C., Møller, P., \& Thomsen, B. 2005, in Astrophysics and Space Science Library, Vol. 329, Starbursts: From 30 Doradus to Lyman Break Galaxies, ed. R. de Grijs \& R. M. González Delgado, 293

Fynbo, J. P. U., Ledoux, C., Möller, P., Thomsen, B., \& Burud, I. 2003b, A\&A, 407, 147 
Fynbo, J. P. U., Möller, P., Thomsen, B., Hjorth, J., Gorosabel, J., Andersen, M. I., Egholm, M. P., Holland, S., Jensen, B. L., Pedersen, H., \& Weidinger, M. 2002, A\&A, 388, 425

Fynbo, J. P. U., Prochaska, J. X., Sommer-Larsen, J., Dessauges-Zavadsky, M., \& Møller, P. 2008b, ApJ, 683, 321

Fynbo, J. P. U., Watson, D., Thöne, C. C., Sollerman, J., Bloom, J. S., Davis, T. M., Hjorth, J., Jakobsson, P., Jørgensen, U. G., Graham, J. F., Fruchter, A. S., Bersier, D., Kewley, L., Cassan, A., Castro Cerón, J. M., Foley, S., Gorosabel, J., Hinse, T. C., Horne, K. D., Jensen, B. L., Klose, S., Kocevski, D., Marquette, J.-B., Perley, D., Ramirez-Ruiz, E., Stritzinger, M. D., Vreeswijk, P. M., Wijers, R. A. M., Woller, K. G., Xu, D., \& Zub, M. 2006, Nature, 444, 1047

Fynbo, J. U., Gorosabel, J., Dall, T. H., Hjorth, J., Pedersen, H., Andersen, M. I., Møller, P., Holland, S., Smail, I., Kobayashi, N., Rol, E., Vreeswijk, P., Burud, I., Jensen, B. L., Thomsen, B., Henden, A., Vrba, F., Canzian, B., Castro Cerón, J. M., Castro-Tirado, A. J., Cline, T., Goto, M., Greiner, J., Hanski, M. T., Hurley, K., Lund, N., Pursimo, T., Østensen, R., Solheim, J., Tanvir, N., \& Terada, H. 2001, A\&A, 373, 796

Gal-Yam, A., Fox, D. B., Price, P. A., Ofek, E. O., Davis, M. R., Leonard, D. C., Soderberg, A. M., Schmidt, B. P., Lewis, K. M., Peterson, B. A., Kulkarni, S. R., Berger, E., Cenko, S. B., Sari, R., Sharon, K., Frail, D., Moon, D.-S., Brown, P. J., Cucchiara, A., Harrison, F., Piran, T., Persson, S. E., McCarthy, P. J., Penprase, B. E., Chevalier, R. A., \& MacFadyen, A. I. 2006, Nature, 444, 1053

Galama, T. J., Vreeswijk, P. M., van Paradijs, J., Kouveliotou, C., Augusteijn, T., Böhnhardt, H., Brewer, J. P., Doublier, V., Gonzalez, J.-F., Leibundgut, B., Lidman, C., Hainaut, O. R., Patat, F., Heise, J., in't Zand, J., Hurley, K., Groot, P. J., Strom, R. G., Mazzali, P. A., Iwamoto, K., Nomoto, K., Umeda, H., Nakamura, T., Young, T. R., Suzuki, T., Shigeyama, T., Koshut, T., Kippen, M., Robinson, C., de Wildt, P., Wijers, R. A. M. J., Tanvir, N., Greiner, J., Pian, E., Palazzi, E., Frontera, F., Masetti, N., Nicastro, L., Feroci, M., Costa, E., Piro, L., Peterson, B. A., Tinney, C., Boyle, B., Cannon, R., Stathakis, R., Sadler, E., Begam, M. C., \& Ianna, P. 1998, Nature, 395, 670

Gehrels, N., Chincarini, G., Giommi, P., Mason, K. O., Nousek, J. A., Wells, A. A., White, N. E., Barthelmy, S. D., Burrows, D. N., Cominsky, L. R., Hurley, K. C., Marshall, F. E., Mészáros, P., Roming, P. W. A., Angelini, L., Barbier, L. M., Belloni, T., Campana, S., Caraveo, P. A., Chester, M. M., Citterio, O., Cline, T. L., Cropper, 
M. S., Cummings, J. R., Dean, A. J., Feigelson, E. D., Fenimore, E. E., Frail, D. A., Fruchter, A. S., Garmire, G. P., Gendreau, K., Ghisellini, G., Greiner, J., Hill, J. E., Hunsberger, S. D., Krimm, H. A., Kulkarni, S. R., Kumar, P., Lebrun, F., LloydRonning, N. M., Markwardt, C. B., Mattson, B. J., Mushotzky, R. F., Norris, J. P., Osborne, J., Paczynski, B., Palmer, D. M., Park, H.-S., Parsons, A. M., Paul, J., Rees, M. J., Reynolds, C. S., Rhoads, J. E., Sasseen, T. P., Schaefer, B. E., Short, A. T., Smale, A. P., Smith, I. A., Stella, L., Tagliaferri, G., Takahashi, T., Tashiro, M., Townsley, L. K., Tueller, J., Turner, M. J. L., Vietri, M., Voges, W., Ward, M. J., Willingale, R., Zerbi, F. M., \& Zhang, W. W. 2004, ApJ, 611, 1005

Goad, M. R., Tyler, L. G., Beardmore, A. P., Evans, P. A., Rosen, S. R., Osborne, J. P., Starling, R. L. C., Marshall, F. E., Yershov, V., Burrows, D. N., Gehrels, N., Roming, P. W. A., Moretti, A., Capalbi, M., Hill, J. E., Kennea, J., Koch, S., \& vanden Berk, D. 2007, A\&A, 476, 1401

Greiner, J., Krühler, T., Klose, S., Afonso, P., Clemens, C., Filgas, R., Hartmann, D. H., Küpcü Yoldaş, A., Nardini, M., Olivares E., F., Rau, A., Rossi, A., Schady, P., \& Updike, A. 2011, A\&A, 526, A30

Grupe, D., Brown, P. J., Cummings, J., Zhang, B., Retter, A., Burrows, D. N., Boyd, P. T., Capalbi, M., Gehrels, N., Holland, S. T., Mészáros, P., Nousek, J. A., Kennea, J. A., O’Brien, P., Osborne, J., Pagani, C., Racusin, J. L., Roming, P., \& Schady, P. 2006, ApJ, 645, 464

Grupe, D., Gronwall, C., Wang, X.-Y., Roming, P. W. A., Cummings, J., Zhang, B., Mészáros, P., Trigo, M. D., O’Brien, P. T., Page, K. L., Beardmore, A., Godet, O., vanden Berk, D. E., Brown, P. J., Koch, S., Morris, D., Stroh, M., Burrows, D. N., Nousek, J. A., McMath Chester, M., Immler, S., Mangano, V., Romano, P., Chincarini, G., Osborne, J., Sakamoto, T., \& Gehrels, N. 2007, ApJ, 662, 443

Guidorzi, C. 2005, MNRAS, 364, 163

Guidorzi, C., Barthelmy, S. D., Evans, P. A., Gehrels, N., Gronwall, C., Kennea, J. A., Krimm, H. A., Mangano, V., Moretti, A., Page, K. L., Palmer, D. M., Romano, P., \& Vergani, S. D. 2006, GRB Coordinates Network, 5575, 1

Hjorth, J., Sollerman, J., Møller, P., Fynbo, J. P. U., Woosley, S. E., Kouveliotou, C., Tanvir, N. R., Greiner, J., Andersen, M. I., Castro-Tirado, A. J., Castro Cerón, J. M., Fruchter, A. S., Gorosabel, J., Jakobsson, P., Kaper, L., Klose, S., Masetti, N., Pedersen, H., Pedersen, K., Pian, E., Palazzi, E., Rhoads, J. E., Rol, E., van den 
Heuvel, E. P. J., Vreeswijk, P. M., Watson, D., \& Wijers, R. A. M. J. 2003, Nature, 423,847

Holland, S. T., Boyd, P. T., Gorosabel, J., Hjorth, J., Schady, P., Thomsen, B., Augusteijn, T., Blustin, A. J., Breeveld, A., De Pasquale, M., Fynbo, J. P. U., Gehrels, N., Gronwall, C., Hunsberger, S., Ivanushkina, M., Landsman, W., Laursen, P., McGowan, K., Mangano, V., Markwardt, C. B., Marshall, F., Mason, K. O., Moretti, A., Page, M. J., Poole, T., Roming, P., Rosen, S., \& Still, M. 2007, AJ, 133, 122

Hunt, L., Palazzi, E., Rossi, A., Savaglio, S., Cresci, G., Klose, S., Michałowski, M., \& Pian, E. 2011, ApJ, 736, L36

Jakobsson, P., Björnsson, G., Fynbo, J. P. U., Jóhannesson, G., Hjorth, J., Thomsen, B., Møller, P., Watson, D., Jensen, B. L., Östlin, G., Gorosabel, J., \& Gudmundsson, E. H. 2005, MNRAS, 362, 245

Jakobsson, P., Fynbo, J. P. U., Ledoux, C., Vreeswijk, P., Kann, D. A., Hjorth, J., Priddey, R. S., Tanvir, N. R., Reichart, D., Gorosabel, J., Klose, S., Watson, D., Sollerman, J., Fruchter, A. S., de Ugarte Postigo, A., Wiersema, K., Björnsson, G., Chapman, R., Thöne, C. C., Pedersen, K., \& Jensen, B. L. 2006a, A\&A, 460, L13

Jakobsson, P., Hjorth, J., Fynbo, J. P. U., Gorosabel, J., \& Jaunsen, A. O. 2008, in The Eleventh Marcel Grossmann Meeting On Recent Developments in Theoretical and Experimental General Relativity, Gravitation and Relativistic Field Theories, ed. H. Kleinert, R. T. Jantzen, \& R. Ruffini (Singapore: World Scientific), 2019

Jakobsson, P., Hjorth, J., Fynbo, J. P. U., Watson, D., Pedersen, K., Björnsson, G., \& Gorosabel, J. 2004, ApJ, 617, L21

Jakobsson, P., Hjorth, J., Malesani, D., Chapman, R., Fynbo, J. P. U., Tanvir, N. R., Milvang-Jensen, B., Vreeswijk, P. M., Letawe, G., \& Starling, R. L. C. 2012, ApJ, 752,62

Jakobsson, P., Levan, A., Fynbo, J. P. U., Priddey, R., Hjorth, J., Tanvir, N., Watson, D., Jensen, B. L., Sollerman, J., Natarajan, P., Gorosabel, J., Castro Cerón, J. M., Pedersen, K., Pursimo, T., Árnadóttir, A. S., Castro-Tirado, A. J., Davis, C. J., Deeg, H. J., Fiuza, D. A., Mikolaitis, S., \& Sousa, S. G. 2006b, A\&A, 447, 897

Jakobsson, P., Malesani, D., Fynbo, J. P. U., Hjorth, J., \& Milvang-Jensen, B. 2009, in AIP Conf. Proc. 1133, Gamma-Ray Burst: Sixth Huntsville Symposium, ed. C. Meegan, C. Kouveliotou, \& N. Gehrels (Melville, NY: AIP), 455 
Jakobsson, P., Malesani, D., Fynbo, J. P. U., Hjorth, J., Vreeswijk, P. M., \& Tanvir, N. R. 2007, GRB Coordinates Network, 6997, 1

Jakobsson, P., Malesani, D., Hjorth, J., Fynbo, J. P. U., \& Milvang-Jensen, B. 2011a, Advances in Space Research, 47, 1416

—. 2011b, Astronomische Nachrichten, 332, 276

Jakobsson, P., Malesani, D., Hjorth, J., Fynbo, J. P. U., \& Milvang-Jensen, B. 2011c, in AIP Conf. Proc. 1358, Gamma-Ray Bursts 2010 ed. J. E. McEnery, J. L. Racusin, \& N. Gehrels (Melville, NY: AIP), 265

Jaunsen, A. O., Rol, E., Watson, D. J., Malesani, D., Fynbo, J. P. U., Milvang-Jensen, B., Hjorth, J., Vreeswijk, P. M., Ovaldsen, J.-E., Wiersema, K., Tanvir, N. R., Gorosabel, J., Levan, A. J., Schirmer, M., \& Castro-Tirado, A. J. 2008, ApJ, 681, 453

Jelínek, M., Prouza, M., Kubánek, P., Hudec, R., Nekola, M., Řídký, J., Grygar, J., Boháčová, M., Castro-Tirado, A. J., Gorosabel, J., Hrabovský, M., Mandát, D., Nosek, D., Nožka, L., Palatka, M., Pandey, S. B., Pech, M., Schovánek, P., Šmída, R., Trávníček, P., de Ugarte Postigo, A., \& Vítek, S. 2006, A\&A, 454, L119

Jensen, B. L., Hjorth, J., Fynbo, J., \& Naranen, J. 2006, GRB Coordinates Network, 5203, 1

Kamble, A., Misra, K., Bhattacharya, D., \& Sagar, R. 2009, MNRAS, 394, 214

Kasliwal, M. M., Cenko, S. B., Kulkarni, S. R., Cameron, P. B., Nakar, E., Ofek, E. O., Rau, A., Soderberg, A. M., Campana, S., Bloom, J. S., Perley, D. A., Pollack, L. K., Barthelmy, S., Cummings, J., Gehrels, N., Krimm, H. A., Markwardt, C. B., Sato, G., Chandra, P., Frail, D., Fox, D. B., Price, P. A., Berger, E., Grebenev, S. A., Krivonos, R. A., \& Sunyaev, R. A. 2008, ApJ, 678, 1127

Kawai, N., Kosugi, G., Aoki, K., Yamada, T., Totani, T., Ohta, K., Iye, M., Hattori, T., Aoki, W., Furusawa, H., Hurley, K., Kawabata, K. S., Kobayashi, N., Komiyama, Y., Mizumoto, Y., Nomoto, K., Noumaru, J., Ogasawara, R., Sato, R., Sekiguchi, K., Shirasaki, Y., Suzuki, M., Takata, T., Tamagawa, T., Terada, H., Watanabe, J., Yatsu, Y., \& Yoshida, A. 2006, Nature, 440, 184

Kennea, J. A., Burrows, D. N., \& Page, K. 2005, GRB Coordinates Network, 3827, 1

Kouveliotou, C., Meegan, C. A., Fishman, G. J., Bhat, N. P., Briggs, M. S., Koshut, T. M., Paciesas, W. S., \& Pendleton, G. N. 1993, ApJ, 413, L101 
Krühler, T., Greiner, J., Schady, P., Savaglio, S., Afonso, P. M. J., Clemens, C., Elliott, J., Filgas, R., Gruber, D., Kann, D. A., Klose, S., Küpcü-Yoldaş, A., McBreen, S., Olivares, F., Pierini, D., Rau, A., Rossi, A., Nardini, M., Nicuesa Guelbenzu, A., Sudilovsky, V., \& Updike, A. C. 2011, A\&A, 534, A108

Krühler, T., Malesani, D., Milvang-Jensen, B., Fynbo, J. P. U., Hjorth, J., Jakobsson, P., Levan, A. J., Sparre, M., Tanvir, N. R., \& Watson, D. J. 2012, ApJ, in press (arXiv:1205.4036)

Kuin, N. P. M., Grupe, D., \& Brown, P. 2007, GRB Coordinates Network, 6238, 1

Landsman, W., Marshall, F. E., \& Stroh, M. C. 2007, GRB Coordinates Network, 6504, 1

Landsman, W., \& Pagani, C. 2007, GRB Coordinates Network, 6378, 1

Laursen, P., Sommer-Larsen, J., \& Andersen, A. C. 2009, ApJ, 704, 1640

Le Floc'h, E., Duc, P.-A., Mirabel, I. F., Sanders, D. B., Bosch, G., Diaz, R. J., Donzelli, C. J., Rodrigues, I., Courvoisier, T. J.-L., Greiner, J., Mereghetti, S., Melnick, J., Maza, J., \& Minniti, D. 2003, A\&A, 400, 499

Levan, A., Beardmore, A., Page, K., Osborne, J., Rol, E., Barthelmy, S., Cannizzo, J., Cominsky, L., Krimm, H., Markwardt, C., Palmer, D., Sakamoto, T., Gronwall, C., Burrows, D., Roming, P., Kennea, J., \& Gehrels, N. 2005, GRB Coordinates Network, 3613,1

Levan, A., Fruchter, A., Rhoads, J., Mobasher, B., Tanvir, N., Gorosabel, J., Rol, E., Kouveliotou, C., Dell'Antonio, I., Merrill, M., Bergeron, E., Castro Cerón, J. M., Masetti, N., Vreeswijk, P., Antonelli, A., Bersier, D., Castro-Tirado, A., Fynbo, J., Garnavich, P., Holland, S., Hjorth, J., Nugent, P., Pian, E., Smette, A., Thomsen, B., Thorsett, S. E., \& Wijers, R. 2006a, ApJ, 647, 471

Levan, A. J., Jakobsson, P., Hurkett, C., Tanvir, N. R., Gorosabel, J., Vreeswijk, P., Rol, E., Chapman, R., Gehrels, N., O’Brien, P. T., Osborne, J. P., Priddey, R. S., Kouveliotou, C., Starling, R., vanden Berk, D., \& Wiersema, K. 2007, MNRAS, 378, 1439

Levan, A. J., Tanvir, N. R., Rol, E., Fruchter, A., \& Adamson, A. 2006b, GRB Coordinates Network, 5455, 1

Malesani, D., Hjorth, J., Fynbo, J. P. U., Milvang-Jensen, B., Jakobsson, P., \& Jaunsen, A. O. 2009, in AIP Conf. Proc. 1111, Probing Stellar Populations Out to the Distant Universe: CELAFU 2008, ed. G. Giobbi, A. Tornambe, G. Raimondo, M. Limongi, L. A. Antonelli, N. Menci, \& E. Brocato (Melville, NY: AIP), 513 
Malesani, D., Hjorth, J., Fynbo, J. P. U., Milvang-Jensen, B., Jakobsson, P., \& Vreeswijk, P. M. 2010, in Proc. Italian Physical Society, Vol. 102, Gamma Ray Bursts and High Energy Shock Phenomena, ed. G. Chincarini, P. D'Avanzo, R. Margutti, R. Salvaterra (Bologna: Italian Physical Society), 303

Malesani, D., Jakobsson, P., Fynbo, J., Stritzinger, M., \& Covino, S. 2006, GRB Coordinates Network, 5350, 1

Malesani, D., Jakobsson, P., Vreeswijk, P. M., Jaunsen, A. O., \& Kann, D. A. 2007, GRB Coordinates Network, 6055, 1

Mangano, V., Holland, S. T., Malesani, D., Troja, E., Chincarini, G., Zhang, B., La Parola, V., Brown, P. J., Burrows, D. N., Campana, S., Capalbi, M., Cusumano, G., Della Valle, M., Gehrels, N., Giommi, P., Grupe, D., Guidorzi, C., Mineo, T., Moretti, A., Osborne, J. P., Pandey, S. B., Perri, M., Romano, P., Roming, P. W. A., \& Tagliaferri, G. 2007, A\&A, 470, 105

Markwardt, C. B., Chester, M. M., Cummings, J. R., Evans, P. A., Gehrels, N., Kennea, J. A., Krimm, H. A., Marshall, F. E., Pagani, C., Page, K. L., Palmer, D. M., Stamatikos, M., \& Troja, E. 2007, GRB Coordinates Network, 6224, 1

Melandri, A., Mundell, C. G., Kobayashi, S., Mottram, C. J., Steele, I. A., Smith, R. J., Carter, D., Bersier, D., \& Bode, M. F. 2006, GRB Coordinates Network, 5804, 1

Melandri, A., Sbarufatti, B., D’Avanzo, P., Salvaterra, R., Campana, S., Covino, S., Vergani, S. D., Nava, L., Ghisellini, G., Ghirlanda, G., Fugazza, D., Mangano, V., Capalbi, M., \& Tagliaferri, G. 2012, MNRAS, 421, 1265

Michałowski, M. J., Hjorth, J., Castro Cerón, J. M., \& Watson, D. 2008, ApJ, 672, 817

Michałowski, M. J., Kamble, A., Hjorth, J., Malesani, D., Reinfrank, R. F., Bonavera, L., Castro Cerón, J. M., Ibar, E., Dunlop, J. S., Fynbo, J. P. U., Garrett, M. A., Jakobsson, P., Kaplan, D. L., Krühler, T., Levan, A. J., Massardi, M., Pal, S., Sollerman, J., Tanvir, N. R., van der Horst, A. J., Watson, D., \& Wiersema, K. 2012, ApJ, 755, 85

Milvang-Jensen, B., Fynbo, J. P. U., Malesani, D., Hjorth, J., Jakobsson, P., \& Møller, P. 2012, ApJ, 756, 25

Mineo, T., Mangano, V., Covino, S., Cusumano, G., La Parola, V., Troja, E., Roming, P., Burrows, D. N., Campana, S., Capalbi, M., Chincarini, G., Gehrels, N., Giommi, P., Hill, J. E., Marshall, F., Moretti, A., O'Brien, P., Page, M., Perri, M., Romano, P., Sbarufatti, B., Sato, G., \& Tagliaferri, G. 2007, A\&A, 469, 663 
Mirabal, N., Halpern, J. P., An, D., Thorstensen, J. R., \& Terndrup, D. M. 2006, ApJ, 643, L99

Modjaz, M., Stanek, K. Z., Garnavich, P. M., Berlind, P., Blondin, S., Brown, W., Calkins, M., Challis, P., Diamond-Stanic, A. M., Hao, H., Hicken, M., Kirshner, R. P., \& Prieto, J. L. 2006, ApJ, 645, L21

Møller, P., Warren, S. J., Fall, S. M., Fynbo, J. U., \& Jakobsen, P. 2002, ApJ, 574, 51

Monet, D. G., Levine, S. E., Canzian, B., Ables, H. D., Bird, A. R., Dahn, C. C., Guetter, H. H., Harris, H. C., Henden, A. A., Leggett, S. K., Levison, H. F., Luginbuhl, C. B., Martini, J., Monet, A. K. B., Munn, J. A., Pier, J. R., Rhodes, A. R., Riepe, B., Sell, S., Stone, R. C., Vrba, F. J., Walker, R. L., Westerhout, G., Brucato, R. J., Reid, I. N., Schoening, W., Hartley, M., Read, M. A., \& Tritton, S. B. 2003, AJ, 125, 984

Moretti, A., Cummings, J., Gehrels, N., Hunsberger, S., Krimm, H., Markwardt, C., Marshall, F., Palmer, D., \& Romano, P. 2005, GRB Coordinates Network, 4051, 1

Morris, D., Pagani, C., Capalbi, M., Kennea, J., \& Burrows, D. 2005, GRB Coordinates Network, 4062, 1

Norris, J., Barbier, L., Barthelmy, S., Boyd, P., Burrows, D., Cummings, J., Gehrels, N., Holland, S. T., Kennea, J., Krimm, H., Marshall, F., Godet, O., Palmer, D., \& Sakamoto, T. 2005, GRB Coordinates Network, 4008, 1

Oates, S. R., Page, M. J., Schady, P., de Pasquale, M., Koch, T. S., Breeveld, A. A., Brown, P. J., Chester, M. M., Holland, S. T., Hoversten, E. A., Kuin, N. P. M., Marshall, F. E., Roming, P. W. A., Still, M., vanden Berk, D. E., Zane, S., \& Nousek, J. A. 2009, MNRAS, 395, 490

Ofek, E. O., Cenko, S. B., Gal-Yam, A., Fox, D. B., Nakar, E., Rau, A., Frail, D. A., Kulkarni, S. R., Price, P. A., Schmidt, B. P., Soderberg, A. M., Peterson, B., Berger, E., Sharon, K., Shemmer, O., Penprase, B. E., Chevalier, R. A., Brown, P. J., Burrows, D. N., Gehrels, N., Harrison, F., Holland, S. T., Mangano, V., McCarthy, P. J., Moon, D.-S., Nousek, J. A., Persson, S. E., Piran, T., \& Sari, R. 2007, ApJ, 662, 1129

Osip, D., Chen, H.-W., \& Prochaska, J. X. 2006, GRB Coordinates Network, 5715, 1

Page, K. L., Willingale, R., Osborne, J. P., Zhang, B., Godet, O., Marshall, F. E., Melandri, A., Norris, J. P., O'Brien, P. T., Pal'shin, V., Rol, E., Romano, P., Starling, R. L. C., Schady, P., Yost, S. A., Barthelmy, S. D., Beardmore, A. P., Cusumano, G., Burrows, D. N., De Pasquale, M., Ehle, M., Evans, P. A., Gehrels, N., Goad, M. R., Golenetskii, 
S., Guidorzi, C., Mundell, C., Page, M. J., Ricker, G., Sakamoto, T., Schaefer, B. E., Stamatikos, M., Troja, E., Ulanov, M., Yuan, F., \& Ziaeepour, H. 2007, ApJ, 663, 1125

Palmer, D., Barbier, L., Barthelmy, S., Cummings, J., Fenimore, E., Gehrels, N., Hullinger, D., Koss, M., Krimm, H., Markwardt, C., Parsons, A., Sakamoto, T., Sato, G., Stamatikos, M., \& Tueller, J. 2006, GRB Coordinates Network, 5208, 1

Pandey, S. B., Castro-Tirado, A. J., McBreen, S., Pérez-Ramírez, M. D., Bremer, M., Guerrero, M. A., Sota, A., Cobb, B. E., Jelínek, M., de Ugarte Postigo, A., Gorosabel, J., Guziy, S., Guidorzi, C., Bailyn, C. D., Muñoz-Darias, T., Gomboc, A., Monfardini, A., Mundell, C. G., Tanvir, N., Levan, A. J., Bhatt, B. C., Sahu, D. K., Sharma, S., Bogdanov, O., \& Combi, J. A. 2006, A\&A, 460, 415

Perley, D. A., Cenko, S. B., Bloom, J. S., Chen, H.-W., Butler, N. R., Kocevski, D., Prochaska, J. X., Brodwin, M., Glazebrook, K., Kasliwal, M. M., Kulkarni, S. R., Lopez, S., Ofek, E. O., Pettini, M., Soderberg, A. M., \& Starr, D. 2009, AJ, 138, 1690

Perri, M., Guetta, D., Antonelli, L. A., Cucchiara, A., Mangano, V., Reeves, J., Angelini, L., Beardmore, A. P., Boyd, P., Burrows, D. N., Campana, S., Capalbi, M., Chincarini, G., Cusumano, G., Giommi, P., Hill, J. E., Holland, S. T., La Parola, V., Mineo, T., Moretti, A., Nousek, J. A., Osborne, J. P., Pagani, C., Romano, P., Roming, P. W. A., Starling, R. L. C., Tagliaferri, G., Troja, E., Vetere, L., \& Gehrels, N. 2007, A\&A, 471, 83

Pian, E., Mazzali, P. A., Masetti, N., Ferrero, P., Klose, S., Palazzi, E., Ramirez-Ruiz, E., Woosley, S. E., Kouveliotou, C., Deng, J., Filippenko, A. V., Foley, R. J., Fynbo, J. P. U., Kann, D. A., Li, W., Hjorth, J., Nomoto, K., Patat, F., Sauer, D. N., Sollerman, J., Vreeswijk, P. M., Guenther, E. W., Levan, A., O'Brien, P., Tanvir, N. R., Wijers, R. A. M. J., Dumas, C., Hainaut, O., Wong, D. S., Baade, D., Wang, L., Amati, L., Cappellaro, E., Castro-Tirado, A. J., Ellison, S., Frontera, F., Fruchter, A. S., Greiner, J., Kawabata, K., Ledoux, C., Maeda, K., Møller, P., Nicastro, L., Rol, E., \& Starling, R. 2006, Nature, 442, 1011

Piranomonte, S., Ward, P. A., Fiore, F., Vergani, S. D., D'Elia, V., Krongold, Y., Nicastro, F., Meurs, E. J. A., Chincarini, G., Covino, S., Della Valle, M., Fugazza, D., Norci, L., Sbordone, L., Stella, L., Tagliaferri, G., Burrows, D. N., Gehrels, N., Goldoni, P., Malesani, D., Mirabel, I. F., Pellizza, L. J., \& Perna, R. 2008, A\&A, 492, 775 
Prochaska, J. X., Chen, H.-W., Bloom, J. S., Dessauges-Zavadsky, M., O'Meara, J. M., Foley, R. J., Bernstein, R., Burles, S., Dupree, A. K., Falco, E., \& Thompson, I. B. 2007, ApJS, 168, 231

Prochaska, J. X., Foley, R. J., Chen, H.-W., Bloom, J. S., Hurley, K., Cooper, M., Guhathakurta, R., \& Li, W. 2005, GRB Coordinates Network, 3971, 1

Racusin, J. L., Barthelmy, S. D., Baumgartner, W. H., Brown, P. J., Burrows, D. N., Cummings, J. R., Evans, P. A., Hunsberger, S. D., Kennea, J. A., Markwardt, C. B., Marshall, F. E., O’Brien, P. T., Palmer, D. M., Sakamoto, T., Starling, R. L. C., Stroh, M. C., \& Ukwatta, T. N. 2008, GRB Coordinates Network, 7264, 1

Robertson, B. E., \& Ellis, R. S. 2012, ApJ, 744, 95

Rol, E., van der Horst, A., Wiersema, K., Patel, S. K., Levan, A., Nysewander, M., Kouveliotou, C., Wijers, R. A. M. J., Tanvir, N., Reichart, D., Fruchter, A. S., Graham, J., Ovaldsen, J.-E., Jaunsen, A. O., Jonker, P., van Ham, W., Hjorth, J., Starling, R. L. C., O’Brien, P. T., Fynbo, J., Burrows, D. N., \& Strom, R. 2007, ApJ, 669, 1098

Roming, P. W. A., Vanden Berk, D., Pal'shin, V., Pagani, C., Norris, J., Kumar, P., Krimm, H., Holland, S. T., Gronwall, C., Blustin, A. J., Zhang, B., Schady, P., Sakamoto, T., Osborne, J. P., Nousek, J. A., Marshall, F. E., Mészáros, P., Golenetskii, S. V., Gehrels, N., Frederiks, D. D., Campana, S., Burrows, D. N., Boyd, P. T., Barthelmy, S., \& Aptekar, R. L. 2006, ApJ, 651, 985

Rossi, A., Klose, S., Ferrero, P., Greiner, J., Arnold, L. A., Gonsalves, E., Hartmann, D. H., Updike, A. C., Kann, D. A., Krühler, T., Palazzi, E., Savaglio, S., Schulze, S., Afonso, P. M. J., Amati, L., Castro-Tirado, A. J., Clemens, C., Filgas, R., Gorosabe, J., Hunt, L. K., Küpcü Yoldas, A., Masetti, N., Nardini, M., Nicuesa Guelbenzu, A., Olivares E., F., Pian, E., Rau, A., Schady, P., Schmidl, S., Yoldas, A., \& de Ugarte Postigo, A. 2012, A\&A, in press (arXiv:1202.1434)

Ruiz-Velasco, A. E., Swan, H., Troja, E., Malesani, D., Fynbo, J. P. U., Starling, R. L. C., Xu, D., Aharonian, F., Akerlof, C., Andersen, M. I., Ashley, M. C. B., Barthelmy, S. D., Bersier, D., Castro Cerón, J. M., Castro-Tirado, A. J., Gehrels, N., Göğüs, E., Gorosabel, J., Guidorzi, C., Güver, T., Hjorth, J., Horns, D., Huang, K. Y., Jakobsson, P., Jensen, B. L., Kızıloğlu, Ü., Kouveliotou, C., Krimm, H. A., Ledoux, C., Levan, A. J., Marsh, T., McKay, T., Melandri, A., Milvang-Jensen, B., Mundell, C. G., O’Brien, P. T., Özel, M., Phillips, A., Quimby, R., Rowell, G., Rujopakarn, W., Rykoff, E. S., Schaefer, B. E., Sollerman, J., Tanvir, N. R., Thöne, C. C., Urata, Y., 
Vestrand, W. T., Vreeswijk, P. M., Watson, D., Wheeler, J. C., Wijers, R. A. M. J., Wren, J., Yost, S. A., Yuan, F., Zhai, M., \& Zheng, W. K. 2007, ApJ, 669, 1

Sakamoto, T., Barbier, L., Barthelmy, S., Boyd, P., Cummings, J., Fenimore, E., Gehrels, N., Hullinger, D., Krimm, H., Markwardt, C., Palmer, D., Parsons, A., Sato, G., Tueller, J., \& Voges, W. 2006, GRB Coordinates Network, 4679, 1

Sakamoto, T., Barthelmy, S. D., Baumgartner, W. H., Cummings, J. R., Fenimore, E. E., Gehrels, N., Krimm, H. A., Markwardt, C. B., Palmer, D. M., Parsons, A. M., Sato, G., Stamatikos, M., Tueller, J., Ukwatta, T. N., \& Zhang, B. 2011, ApJS, 195, 2

Sakamoto, T., Barthelmy, S. D., Burrows, D. N., Chester, M. M., Cummings, J. R., Gehrels, N., Holland, S. T., Immler, S., Kennea, J. A., Krimm, H. A., Mangano, V., Marshall, F. E., McLean, K. M., Pagani, C., Pandey, S. B., Parsons, A. M., Romano, P., Sbarufatti, B., vanden Berk, D. E., \& Ziaeepour, H. 2007, GRB Coordinates Network, 5988, 1

Salvaterra, R., Campana, S., Vergani, S. D., Covino, S., D’Avanzo, P., Fugazza, D., Ghirlanda, G., Ghisellini, G., Melandri, A., Nava, L., Sbarufatti, B., Flores, H., Piranomonte, S., \& Tagliaferri, G. 2012, ApJ, 749, 68

Salvaterra, R., Della Valle, M., Campana, S., Chincarini, G., Covino, S., D'Avanzo, P., Fernández-Soto, A., Guidorzi, C., Mannucci, F., Margutti, R., Thöne, C. C., Antonelli, L. A., Barthelmy, S. D., de Pasquale, M., D'Elia, V., Fiore, F., Fugazza, D., Hunt, L. K., Maiorano, E., Marinoni, S., Marshall, F. E., Molinari, E., Nousek, J., Pian, E., Racusin, J. L., Stella, L., Amati, L., Andreuzzi, G., Cusumano, G., Fenimore, E. E., Ferrero, P., Giommi, P., Guetta, D., Holland, S. T., Hurley, K., Israel, G. L., Mao, J., Markwardt, C. B., Masetti, N., Pagani, C., Palazzi, E., Palmer, D. M., Piranomonte, S., Tagliaferri, G., \& Testa, V. 2009, Nature, 461, 1258

Savaglio, S. 2006, New Journal of Physics, 8, 195

Savaglio, S., Glazebrook, K., \& Le Borgne, D. 2009, ApJ, 691, 182

Sawicki, M., \& Thompson, D. 2006, ApJ, 642, 653

Sbarufatti, B., Barthelmy, S. D., Beardmore, A. P., Brown, P. J., Chester, M. M., Guidorzi, C., Holland, S. T., Kennea, J. A., Markwardt, C. B., Marshall, F. E., Pagani, C., Palmer, D. M., Romano, P., Stamatikos, M., \& Ukwatta, T. N. 2007, GRB Coordinates Network, 6560, 1 
Schady, P., de Pasquale, M., Page, M. J., Vetere, L., Pandey, S. B., Wang, X. Y., Cummings, J., Zhang, B., Zane, S., Breeveld, A., Burrows, D. N., Gehrels, N., Gronwall, C., Hunsberger, S., Markwardt, C., Mason, K. O., Mészáros, P., Norris, J. P., Oates, S. R., Pagani, C., Poole, T. S., Roming, P. W. A., Smith, P. J., \& vanden Berk, D. E. 2007a, MNRAS, 380, 1041

Schady, P., Mason, K. O., Osborne, J. P., Page, M. J., Roming, P. W. A., Still, M., Zhang, B., Blustin, A. J., Boyd, P., Cucchiara, A., Gehrels, N., Gronwall, C., De Pasquale, M., Holland, S. T., Marshall, F. E., McGowan, K. E., \& Nousek, J. A. 2006, ApJ, 643,276

Schady, P., Mason, K. O., Page, M. J., de Pasquale, M., Morris, D. C., Romano, P., Roming, P. W. A., Immler, S., \& vanden Berk, D. E. 2007b, MNRAS, 377, 273

Schady, P., \& Moretti, A. 2006, GRB Coordinates Network, 5294, 1

Schady, P., Page, M. J., Oates, S. R., Still, M., de Pasquale, M., Dwelly, T., Kuin, N. P. M., Holland, S. T., Marshall, F. E., \& Roming, P. W. A. 2010, MNRAS, 401, 2773

Schlegel, D. J., Finkbeiner, D. P., \& Davis, M. 1998, ApJ, 500, 525

Schulze, S., Fynbo, J. P. U., Milvang-Jensen, B., Rossi, A., Jakobsson, P., Ledoux, C., De Cia, A., Kruehler, T., Mehner, A., Bjoernsson, G., Chen, H.-W., Vreeswijk, P. M., Perley, D. A., Hjorth, J., Levan, A. J., Tanvir, N. R., Ellison, S., Moller, P., Worseck, G., Chapman, R., Dall'Aglio, A., \& Letawe, G. 2012a, A\&A, in press (arXiv:1207.6088)

Soderberg, A. M., Berger, E., \& Ofek, E. 2005, GRB Coordinates Network, 4186, 1

Soderberg, A. M., Nakar, E., Cenko, S. B., Cameron, P. B., Frail, D. A., Kulkarni, S. R., Fox, D. B., Berger, E., Gal-Yam, A., Moon, D.-S., Price, P. A., Anderson, G., Schmidt, B. P., Salvo, M., Rich, J., Rau, A., Ofek, E. O., Chevalier, R. A., Hamuy, M., Harrison, F. A., Kumar, P., MacFadyen, A., McCarthy, P. J., Park, H. S., Peterson, B. A., Phillips, M. M., Rauch, M., Roth, M., \& Shectman, S. 2007, ApJ, 661, 982

Sollerman, J., Fynbo, J. P. U., Gorosabel, J., Halpern, J. P., Hjorth, J., Jakobsson, P., Mirabal, N., Watson, D., Xu, D., Castro-Tirado, A. J., Féron, C., Jaunsen, A. O., Jelínek, M., Jensen, B. L., Kann, D. A., Ovaldsen, J. E., Pozanenko, A., Stritzinger, M., Thöne, C. C., de Ugarte Postigo, A., Guziy, S., Ibrahimov, M., Järvinen, S. P., Levan, A., Rumyantsev, V., \& Tanvir, N. 2007, A\&A, 466, 839 
Sollerman, J., Jaunsen, A. O., Fynbo, J. P. U., Hjorth, J., Jakobsson, P., Stritzinger, M., Féron, C., Laursen, P., Ovaldsen, J.-E., Selj, J., Thöne, C. C., Xu, D., Davis, T., Gorosabel, J., Watson, D., Duro, R., Ilyin, I., Jensen, B. L., Lysfjord, N., Marquart, T., Nielsen, T. B., Näränen, J., Schwarz, H. E., Walch, S., Wold, M., \& Östlin, G. 2006, A\&A, 454, 503

Stamatikos, M., Barthelmy, S. D., Burrows, D. N., Capalbi, M., Conciatore, M. L., Gehrels, N., Guidorzi, C., Holland, S. T., Kennea, J. A., Markwardt, C. B., Palmer, D. M., Perri, M., Sakamoto, T., vanden Berk, D. E., \& Ziaeepour, H. 2006, GRB Coordinates Network, 5590, 1

Stanek, K. Z., Matheson, T., Garnavich, P. M., Martini, P., Berlind, P., Caldwell, N., Challis, P., Brown, W. R., Schild, R., Krisciunas, K., Calkins, M. L., Lee, J. C., Hathi, N., Jansen, R. A., Windhorst, R., Echevarria, L., Eisenstein, D. J., Pindor, B., Olszewski, E. W., Harding, P., Holland, S. T., \& Bersier, D. 2003, ApJ, 591, L17

Starling, R. L. C., Vreeswijk, P. M., Ellison, S. L., Rol, E., Wiersema, K., Levan, A. J., Tanvir, N. R., Wijers, R. A. M. J., Tadhunter, C., Rodriguez Zaurin, J., Gonzalez Delgado, R. M., \& Kouveliotou, C. 2005, A\&A, 442, L21

Steidel, C. C., Adelberger, K. L., Shapley, A. E., Pettini, M., Dickinson, M., \& Giavalisco, M. 2003, ApJ, 592, 728

Still, M., Roming, P. W. A., Mason, K. O., Blustin, A., Boyd, P., Breeveld, A., Brown, P., De Pasquale, M., Gronwall, C., Holland, S. T., Hunsberger, S., Ivanushkina, M., James, C., Landsman, W., McGowan, K., Morgan, A., Poole, T., Rosen, S., Schady, P., Zhang, B., Krimm, H., Sakamoto, T., Giommi, P., Goad, M. R., Mangano, V., Page, K., Perri, M., Burrows, D. N., Gehrels, N., \& Nousek, J. 2005, ApJ, 635, 1187

Svensson, K. M., Levan, A. J., Tanvir, N. R., Perley, D. A., Michalowski, M. J., Page, K. L., Bloom, J. S., Cenko, S. B., Hjorth, J., Jakobsson, P., Watson, D., \& Wheatley, P. J. 2012, MNRAS, 421, 25

Tagliaferri, G., Antonelli, L. A., Chincarini, G., Fernández-Soto, A., Malesani, D., Della Valle, M., D’Avanzo, P., Grazian, A., Testa, V., Campana, S., Covino, S., Fiore, F., Stella, L., Castro-Tirado, A. J., Gorosabel, J., Burrows, D. N., Capalbi, M., Cusumano, G., Conciatore, M. L., D'Elia, V., Filliatre, P., Fugazza, D., Gehrels, N., Goldoni, P., Guetta, D., Guziy, S., Held, E. V., Hurley, K., Israel, G. L., Jelínek, M., Lazzati, D., López-Echarri, A., Melandri, A., Mirabel, I. F., Moles, M., Moretti, A., Mason, K. O., Nousek, J., Osborne, J., Pellizza, L. J., Perna, R., Piranomonte, S., Piro, L., de Ugarte Postigo, A., \& Romano, P. 2005, A\&A, 443, L1 
Tanvir, N. R., Barnard, V. E., Blain, A. W., Fruchter, A. S., Kouveliotou, C., Natarajan, P., Ramirez-Ruiz, E., Rol, E., Smith, I. A., Tilanus, R. P. J., \& Wijers, R. A. M. J. 2004, MNRAS, 352, 1073

Tanvir, N. R., Fox, D. B., Levan, A. J., Berger, E., Wiersema, K., Fynbo, J. P. U., Cucchiara, A., Krühler, T., Gehrels, N., Bloom, J. S., Greiner, J., Evans, P. A., Rol, E., Olivares, F., Hjorth, J., Jakobsson, P., Farihi, J., Willingale, R., Starling, R. L. C., Cenko, S. B., Perley, D., Maund, J. R., Duke, J., Wijers, R. A. M. J., Adamson, A. J., Allan, A., Bremer, M. N., Burrows, D. N., Castro-Tirado, A. J., Cavanagh, B., de Ugarte Postigo, A., Dopita, M. A., Fatkhullin, T. A., Fruchter, A. S., Foley, R. J., Gorosabel, J., Kennea, J., Kerr, T., Klose, S., Krimm, H. A., Komarova, V. N., Kulkarni, S. R., Moskvitin, A. S., Mundell, C. G., Naylor, T., Page, K., Penprase, B. E., Perri, M., Podsiadlowski, P., Roth, K., Rutledge, R. E., Sakamoto, T., Schady, P., Schmidt, B. P., Soderberg, A. M., Sollerman, J., Stephens, A. W., Stratta, G., Ukwatta, T. N., Watson, D., Westra, E., Wold, T., \& Wolf, C. 2009, Nature, 461, 1254

Tanvir, N. R., Levan, A. J., Fruchter, A. S., Fynbo, J. P. U., Hjorth, J., Wiersema, K., Bremer, M. N., Rhoads, J., Jakobsson, P., O’Brien, P. T., Stanway, E. R., Bersier, D., Natarajan, P., Greiner, J., Watson, D., Castro-Tirado, A. J., Wijers, R. A. M. J., Starling, R. L. C., Misra, K., Graham, J. F., \& Kouveliotou, C. 2012, ApJ, 754, 46

Tanvir, N. R., Levan, A. J., Rol, E., Starling, R. L. C., Gorosabel, J., Priddey, R. S., Malesani, D., Jakobsson, P., O’Brien, P. T., Jaunsen, A. O., Hjorth, J., Fynbo, J. P. U., Melandri, A., Gomboc, A., Milvang-Jensen, B., Fruchter, A. S., Jarvis, M., Fernandes, C. A. C., \& Wold, T. 2008, MNRAS, 388, 1743

Thoene, C. C., Kann, D. A., \& Augusteijn, T. 2007a, GRB Coordinates Network, 6142, 1

Thoene, C. C., Perley, D. A., Cooke, J., Bloom, J. S., Chen, H.-W., \& Barton, E. 2007b, GRB Coordinates Network, 6741, 1

Thöne, C. C., Fynbo, J. P. U., Östlin, G., Milvang-Jensen, B., Wiersema, K., Malesani, D., Della Monica Ferreira, D., Gorosabel, J., Kann, D. A., Watson, D., Michałowski, M. J., Fruchter, A. S., Levan, A. J., Hjorth, J., \& Sollerman, J. 2008, ApJ, 676, 1151

Thöne, C. C., Kann, D. A., Jóhannesson, G., Selj, J. H., Jaunsen, A. O., Fynbo, J. P. U., Akerlof, C. W., Baliyan, K. S., Bartolini, C., Bikmaev, I. F., Bloom, J. S., Burenin, R. A., Cobb, B. E., Covino, S., Curran, P. A., Dahle, H., Ferrero, A., Foley, S., French, J., Fruchter, A. S., Ganesh, S., Graham, J. F., Greco, G., Guarnieri, A., Hanlon, L., Hjorth, J., Ibrahimov, M., Israel, G. L., Jakobsson, P., Jelínek, M., Jensen, B. L., Jørgensen, U. G., Khamitov, I. M., Koch, T. S., Levan, A. J., Malesani, D., 
Masetti, N., Meehan, S., Melady, G., Nanni, D., Näränen, J., Pakstiene, E., Pavlinsky, M. N., Perley, D. A., Piccioni, A., Pizzichini, G., Pozanenko, A., Roming, P. W. A., Rujopakarn, W., Rumyantsev, V., Rykoff, E. S., Sharapov, D., Starr, D., Sunyaev, R. A., Swan, H., Tanvir, N. R., Terra, F., de Ugarte Postigo, A., Vreeswijk, P. M., Wilson, A. C., Yost, S. A., \& Yuan, F. 2010, A\&A, 523, A70

Totani, T., Kawai, N., Kosugi, G., Aoki, K., Yamada, T., Iye, M., Ohta, K., \& Hattori, T. 2006, PASJ, 58, 485

Trenti, M., Perna, R., Levesque, E. M., Shull, J. M., \& Stocke, J. T. 2012, ApJ, 749, L38

Troja, E., Cusumano, G., O’Brien, P. T., Zhang, B., Sbarufatti, B., Mangano, V., Willingale, R., Chincarini, G., Osborne, J. P., Marshall, F. E., Burrows, D. N., Campana, S., Gehrels, N., Guidorzi, C., Krimm, H. A., La Parola, V., Liang, E. W., Mineo, T., Moretti, A., Page, K. L., Romano, P., Tagliaferri, G., Zhang, B. B., Page, M. J., \& Schady, P. 2007, ApJ, 665, 599

van Paradijs, J., Kouveliotou, C., \& Wijers, R. A. M. J. 2000, ARA\&A, 38, 379

Verhamme, A., Schaerer, D., \& Maselli, A. 2006, A\&A, 460, 397

Watson, D., Fynbo, J. P. U., Ledoux, C., Vreeswijk, P., Hjorth, J., Smette, A., Andersen, A. C., Aoki, K., Augusteijn, T., Beardmore, A. P., Bersier, D., Castro Cerón, J. M., D’Avanzo, P., Diaz-Fraile, D., Gorosabel, J., Hirst, P., Jakobsson, P., Jensen, B. L., Kawai, N., Kosugi, G., Laursen, P., Levan, A., Masegosa, J., Näränen, J., Page, K. L., Pedersen, K., Pozanenko, A., Reeves, J. N., Rumyantsev, V., Shahbaz, T., Sharapov, D., Sollerman, J., Starling, R. L. C., Tanvir, N., Torstensson, K., \& Wiersema, K. 2006, ApJ, 652, 1011

Wiersema, K., Savaglio, S., Vreeswijk, P. M., Ellison, S. L., Ledoux, C., Yoon, S.-C., Møller, P., Sollerman, J., Fynbo, J. P. U., Pian, E., Starling, R. L. C., \& Wijers, R. A. M. J. 2007, A\&A, 464, 529

Wijers, R. A. M. J., Bloom, J. S., Bagla, J. S., \& Natarajan, P. 1998, MNRAS, 294, L13

Wolfe, A. M., Gawiser, E., \& Prochaska, J. X. 2005, ARA\&A, 43, 861

Xu, D., Starling, R. L. C., Fynbo, J. P. U., Sollerman, J., Yost, S., Watson, D., Foley, S., O’Brien, P. T., \& Hjorth, J. 2009, ApJ, 696, 971

Yanagisawa, K., Toda, H., \& Kawai, N. 2006, GRB Coordinates Network, 4510, 1 
Ziaeepour, H., Barthelmy, S. D., Beardmore, A. P., Burrows, D. N., Evans, P. A., Page, K. L., \& Troja, E. 2007, GRB Coordinates Network, 6639, 1

Ziaeepour, H., Burrows, D. N., Cummings, J. R., Oates, S. R., Pagani, C., Page, K. L., Palmer, D. M., Sato, G., Stamatikos, M., Starling, R. L. C., \& vanden Berk, D. E. 2006a, GRB Coordinates Network, 5691, 1

Ziaeepour, H., Holland, S. T., Boyd, P. T., Page, K., Oates, S., Markwardt, C. B., Mészáros, P., Gehrels, N., Marshall, F. E., Cummings, J., \& Goad, M. 2008, MNRAS, 385, 453

Ziaeepour, H. Z., Barthelmy, S. D., Cummings, J. R., Gehrels, N., Guidorzi, C., Kennea, J. A., Krimm, H. A., Markwardt, C. B., Marshall, F. E., McLean, K. M., Page, K. L., Page, M. J., Palmer, D. M., \& Sakamoto, T. 2006b, GRB Coordinates Network, 5398, 1 


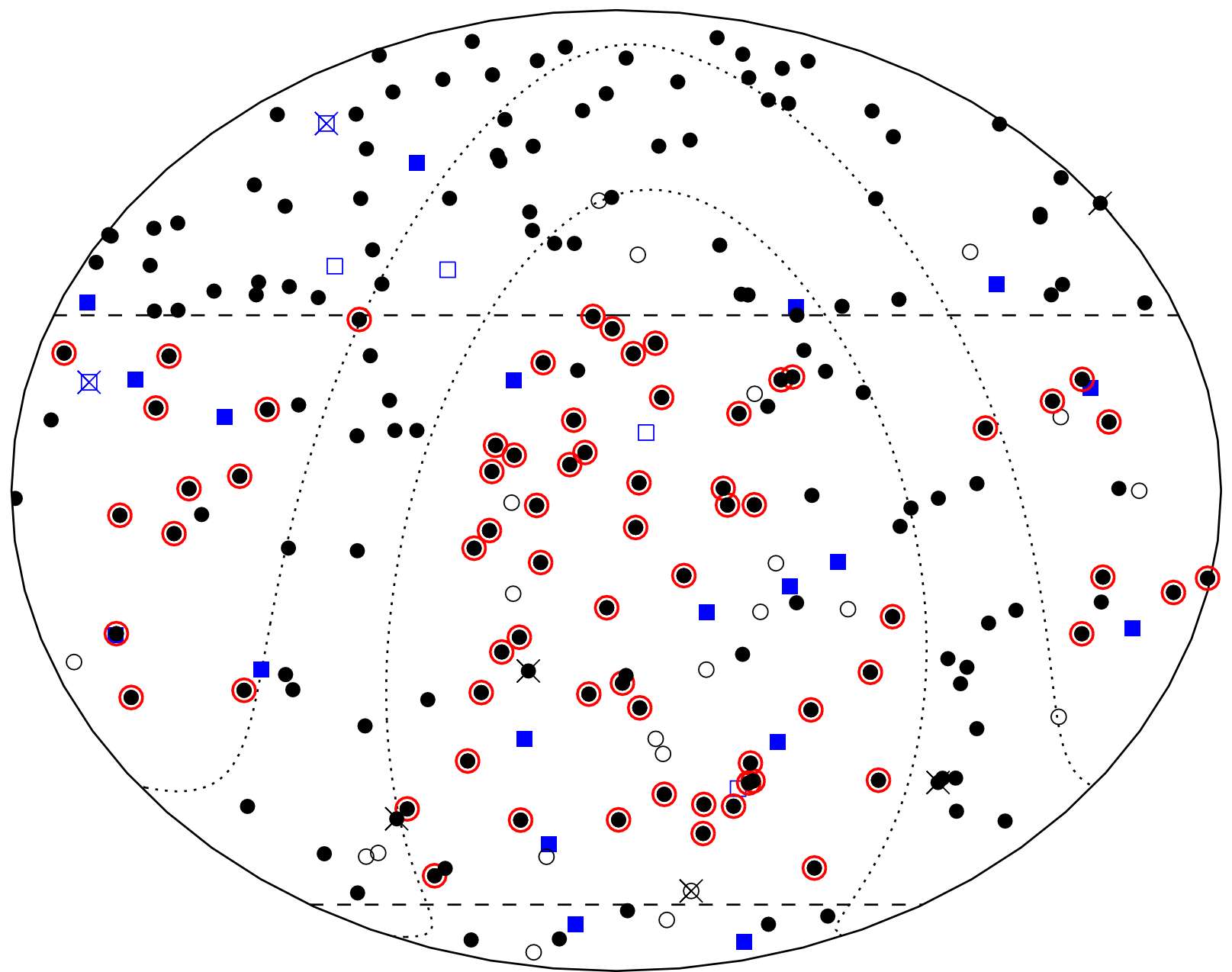

Fig. 1.- All-sky map (Mollweid projection) of the 236 Swift GRBs which occurred between 2005 March 1 and 2007 August 10 (Criterion 7). Filled circles: GRBs with Swift/XRT detection. Filled circles, encircled in red: GRBs obeying all our sample selection Criteria 1-9. Empty circles: GRBs with no Swift/XRT detection (excluded by Criterion 3). Blue squares: GRBs classified as short (excluded by Criterion 2). Crosses: nontriggered GRBs (excluded by Criterion 1). The declination cuts are shown with dashed lines $\left(-70^{\circ}\right.$ and $+27^{\circ}$; Criterion 8) and the region with Galactic latitude $|b|<15^{\circ}$ is shown with the dotted curves, which roughly corresponds to the sample selection criterion $A_{V} \leqslant 0.5 \mathrm{mag}$ (Criterion 4). 


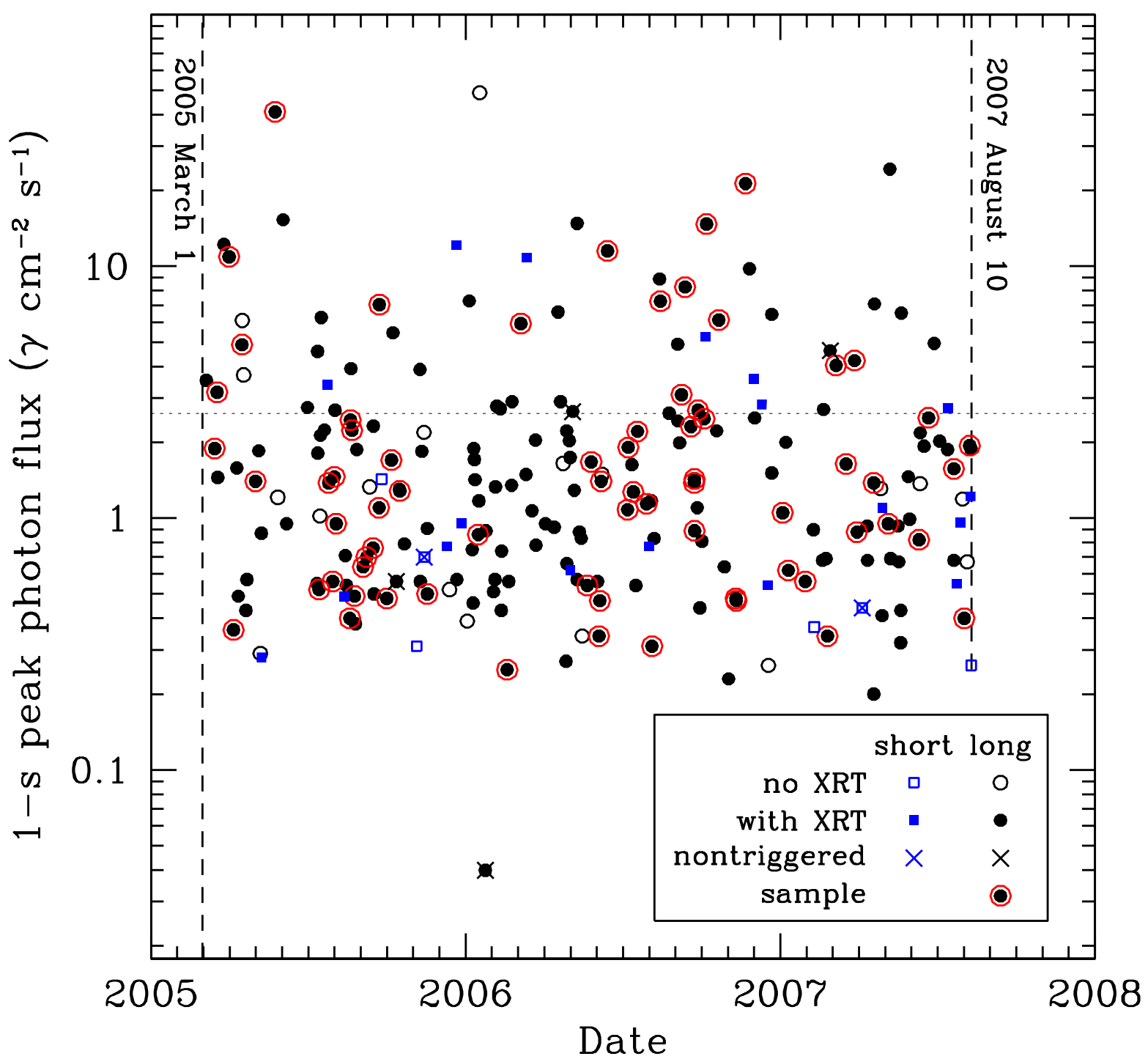

Fig. 2.- 1-s Swift/BAT peak photon flux (15-150 keV) for all Swift GRBs observed during the time span of the TOUGH survey. Empty circles: long bursts without an X-ray afterglow. Filled circles: long GRBs with Swift/XRT detection. Filled, encircled circles: GRBs obeying all our sample selection Criteria 1-9. Squares: GRBs classified as short $\left(T_{90}<2\right.$ s). Crosses: nontriggered GRBs. There seems to be a constant and uniform detection level as a function of time (the very faint system at $0.04 \gamma \mathrm{cm}^{-2} \mathrm{~s}^{-1}$ is the unusual GRB 060123; Cummings et al. 2006). The vertical dashed lines bracket the selected time span for the sample. The horizontal dotted line is the peak flux limit adopted by Salvaterra et al. (2012). 


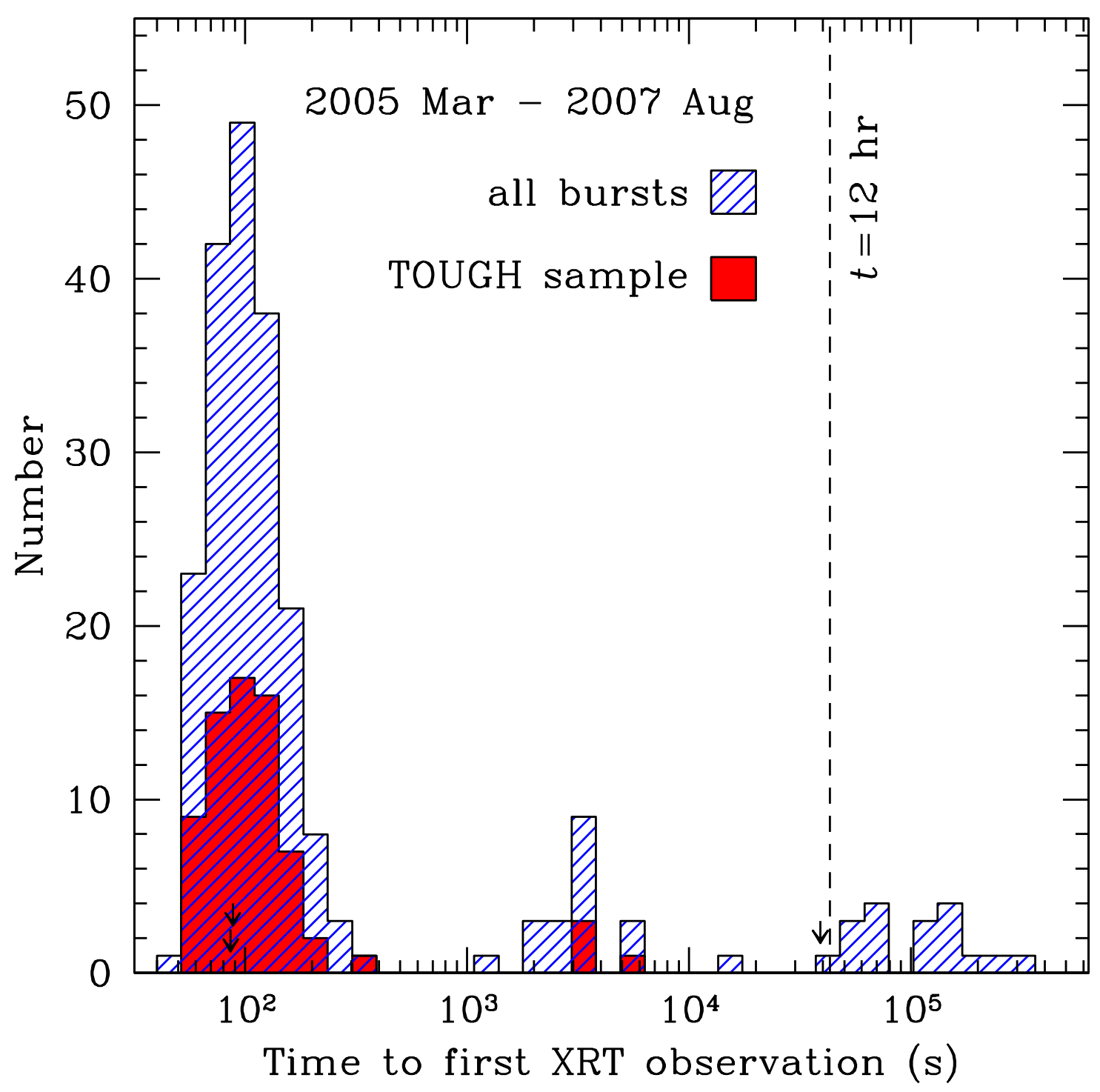

Fig. 3.- Distribution of times post-trigger to the first XRT observation for all Swift GRBs happening between 2005 March 1 and 2007 August 10. For 12 bursts, the XRT was not repointed. The fraction constituting the TOUGH sample is indicated in red. The vertical dashed line marks the sample limit $t \leqslant 12 \mathrm{hr}$, which is fulfilled by $92 \%$ of the repointed bursts $(207 / 224)$. Three GRBs $\left(050603,060223 \mathrm{~A}\right.$, and 070810A) have $t_{\mathrm{XRT}}<12 \mathrm{hr}$ but the notice came later than $12 \mathrm{hr}$. These GRBs are indicated by arrows in the histogram. 


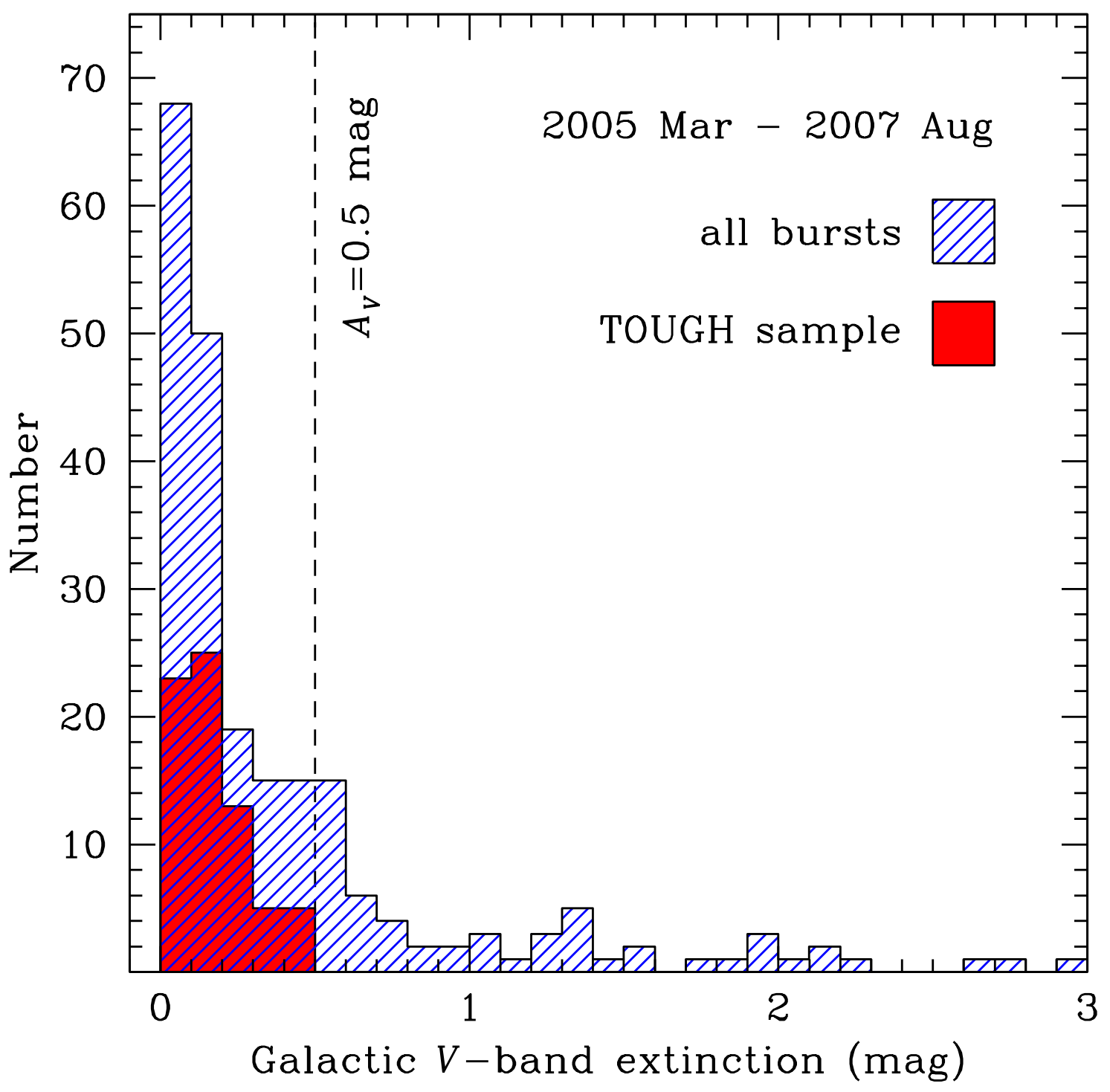

Fig. 4.- Distribution of Galactic foreground extinction for all Swift GRBs detected between 2005 March 1 and 2007 August 10 (13 bursts have 3 mag $<A_{V}<17$ mag and are not shown in the figure). The fraction constituting the TOUGH sample is indicated in red. The vertical dashed line marks the sample limit $A_{V} \leqslant 0.5$ mag. Seventy-one percent of all bursts $(167 / 236)$ have $A_{V} \leqslant 0.5 \mathrm{mag}$. 


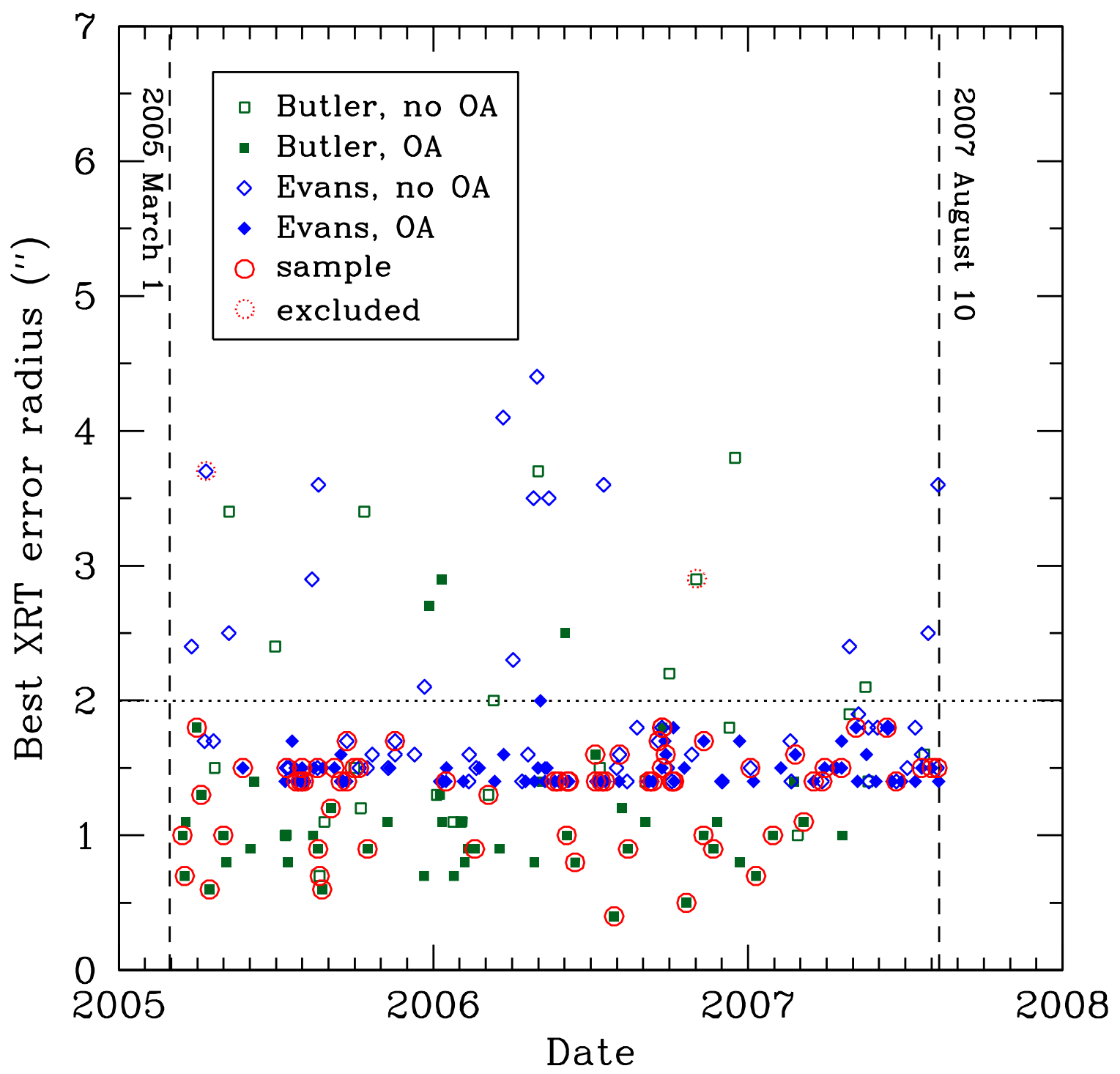

Fig. 5. - Swift/XRT error radius (90\% encircled likelihood) as a function of date for GRBs detected during the time span of the TOUGH survey. Filled and empty points indicate bursts with and without a detected optical/NIR afterglow (OA), respectively. Green squares indicate positions from the Butler (2007b) catalog, while blue diamonds are from the UVOTenhanced list (Goad et al. 2007; Evans et al. 2009). Our sample targets are circled in red. During the period 2005 March 1 through 2007 August 10 (marked by vertical dashed lines), $89 \%$ of the GRBs have an XRT error circle radius $\leqslant 2^{\prime \prime}$ (horizontal dotted line). The two dotted circles indicate the two bursts that are excluded only by Criterion $9\left(r \leqslant 2^{\prime \prime}\right.$; see Figure (6). The average error radius is 1 .'7. 


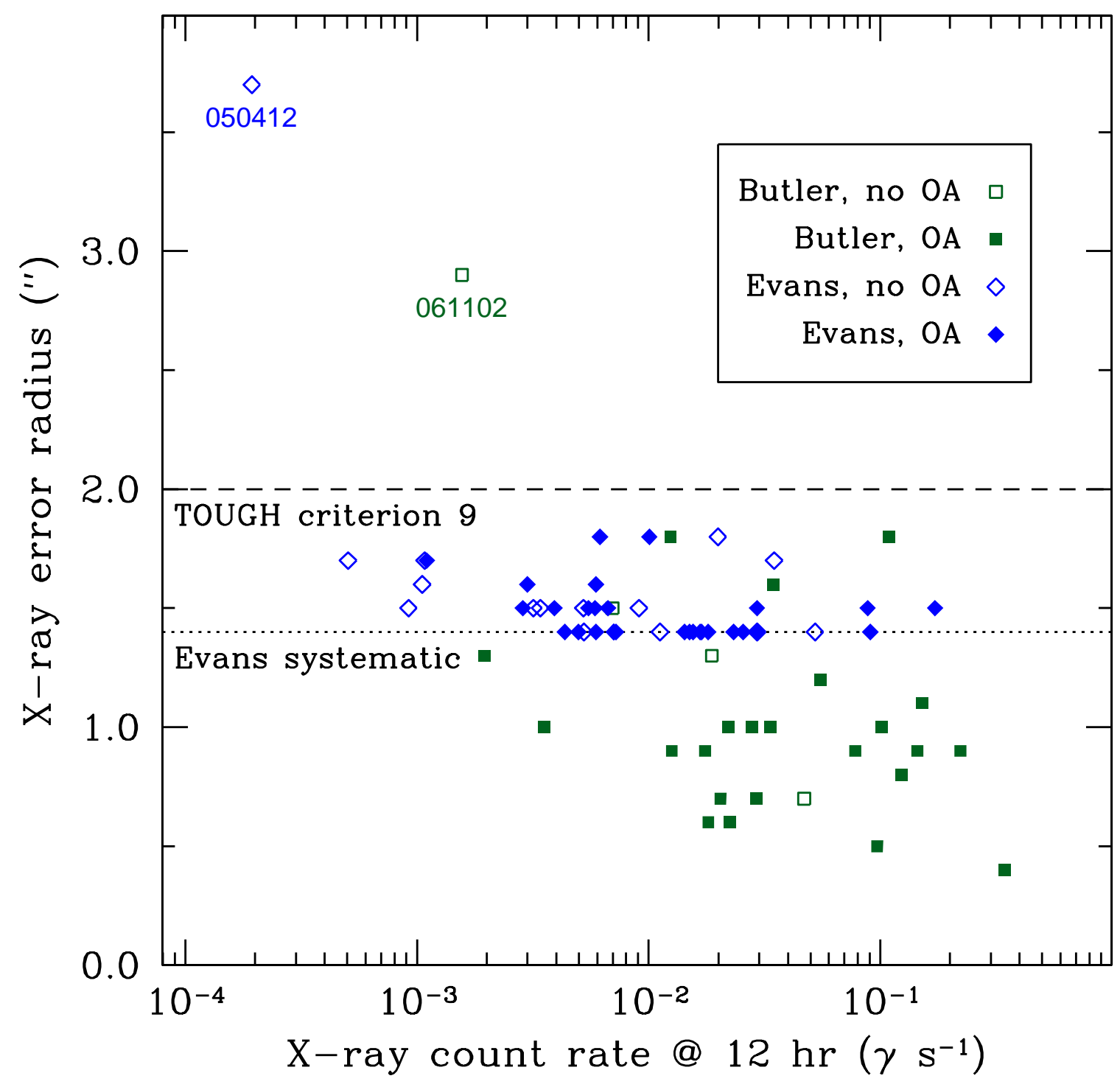

Fig. 6. - X-ray error radius vs. the Swift/XRT count rate at $12 \mathrm{hr}(0.3-10 \mathrm{keV})$. Only bursts which "survive" Criteria 1-8 are shown. Filled and empty points indicate bursts with and without a detected optical/NIR afterglow (OA), respectively. The horizontal dashed line indicates the TOUGH constraint set by Criterion 9. Notice the flat distribution, which implies that we only minimally bias our sample. Indeed, only two GRBs are excluded. The dotted line indicates the systematic error contributing to the UVOT-enhanced uncertainties (Evans et al. 2009). 


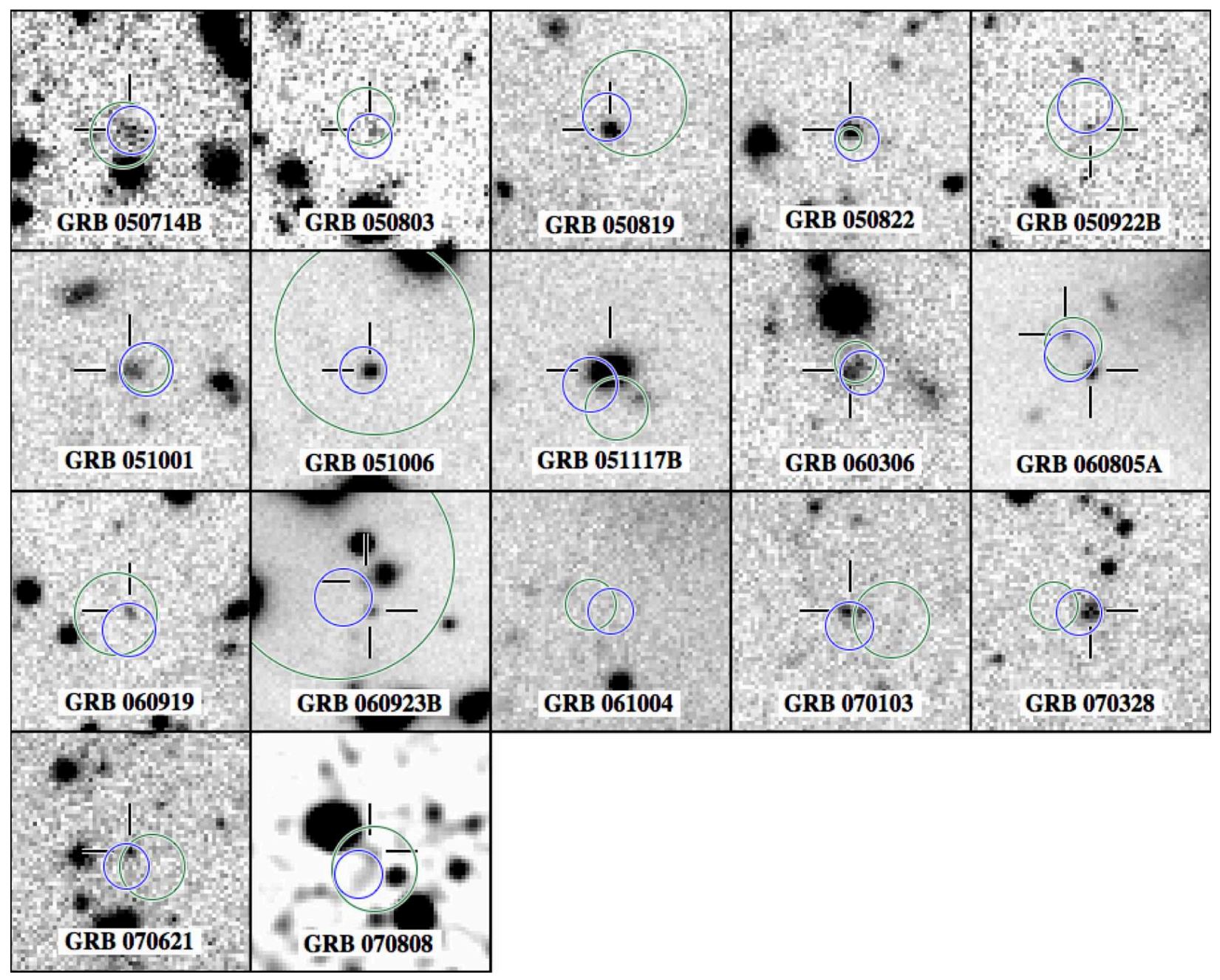

Fig. 7.- Mosaic of the fields of 17 XRT-localized GRBs with no optical/NIR afterglow. Each panel, showing the $R$-band image, is $15^{\prime \prime} \times 15^{\prime \prime}$ in size (north is up and east is left). Blue and green circles identify the XRT positions by Evans et al. (2009) and Butler (2007b), respectively. 


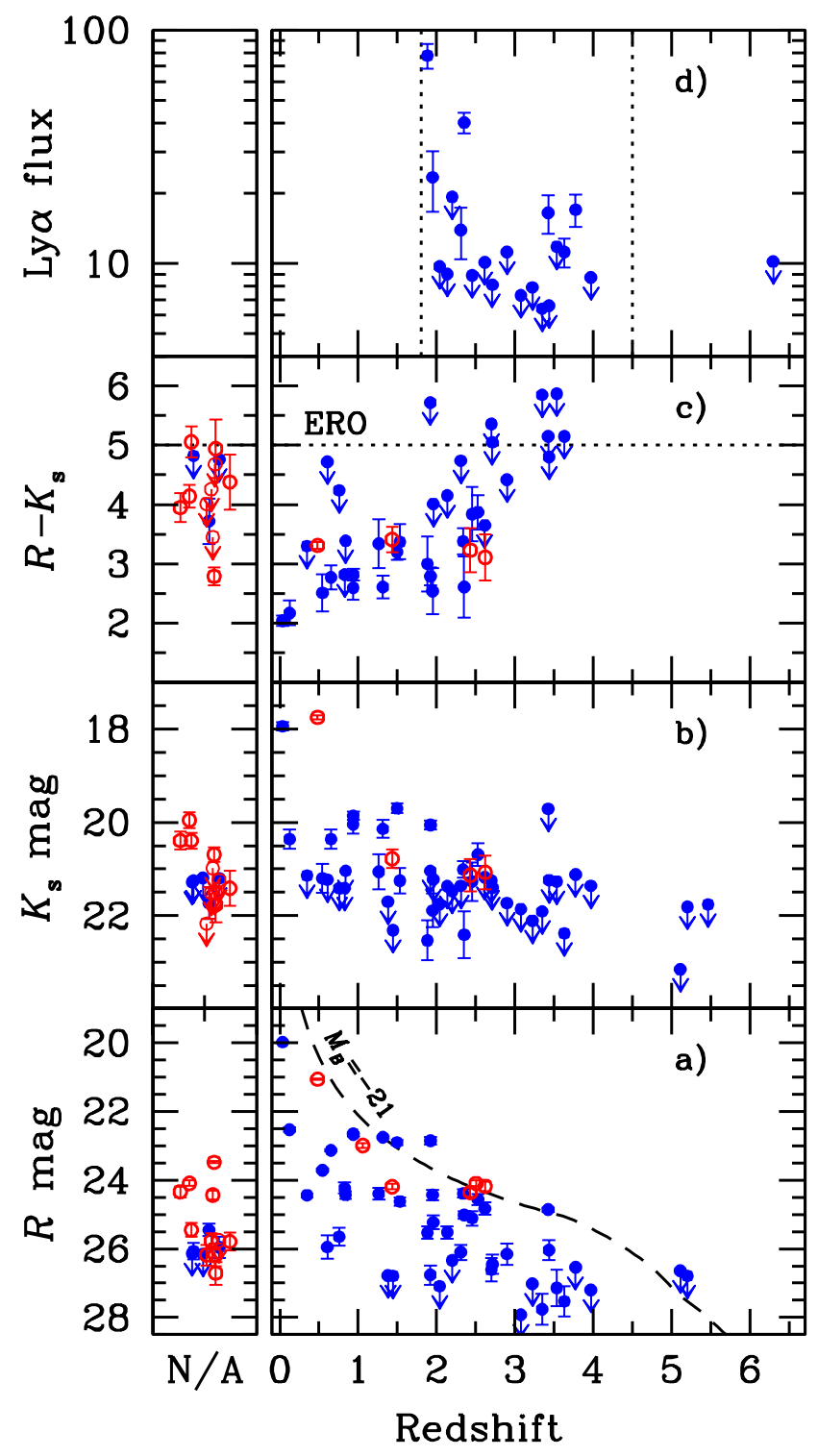

Fig. 8. - TOUGH sample properties as a function of redshift. Optically localized systems are shown as blue filled circles, XRT localized systems are shown as open red circles. Upper limits are shown as arrows. Hosts without a reported redshift are plotted on the left side of the diagram. (a) $R$-band host magnitudes (Vega system). The dashed curve corresponds to the observed $R$-band magnitude for an $M_{B}=-21$ (Vega) object assuming $F_{\nu} \propto \nu^{-0.5}$ and IGM opacity as described by Dall'Aglio et al. (2008). (b) $K_{s}$-band host magnitudes (Vega system). (c) $R-K_{s}$ colors. The ERO limit of $R-K_{s}=5$ is shown as a dotted line. Two EROs are detected. (d) Ly $\alpha$ detections and upper limits from Milvang-Jensen et al. (2012). The upper limit reported for GRB 050904 at $z=6.30$ (Totani et al. 2006) was adjusted to be comparable with those reported in Milvang-Jensen et al. (2012). The Ly $\alpha$ fluxes are in units of $10^{-18} \mathrm{erg} \mathrm{cm}^{-2} \mathrm{~s}^{-1}$. The vertical dotted lines mark the redshift interval where Ly $\alpha$ was searched in our data. 


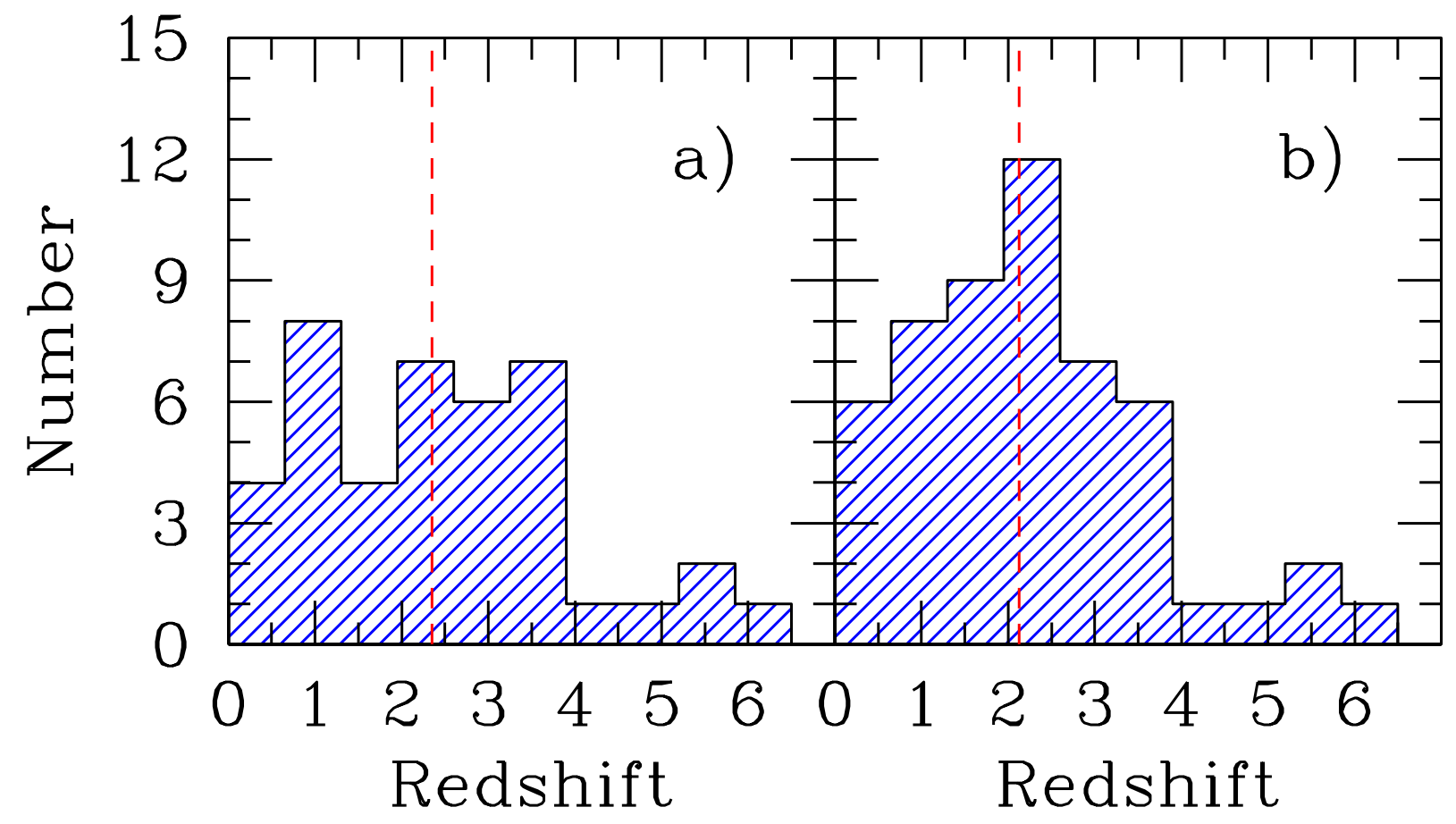

Fig. 9.- Redshift distribution of the TOUGH sample. In both panels the dashed vertical line indicates the median redshift. (a) Without considering the TOUGH observations, 38 redshifts were considered secure with a median redshift of $z=2.35$. (b) Our TOUGH spectroscopic observations add 15 new redshifts and demonstrate that three redshifts reported in the literature are erroneous (Jakobsson et al. 2012). Here the median redshift is $z=2.14$. 


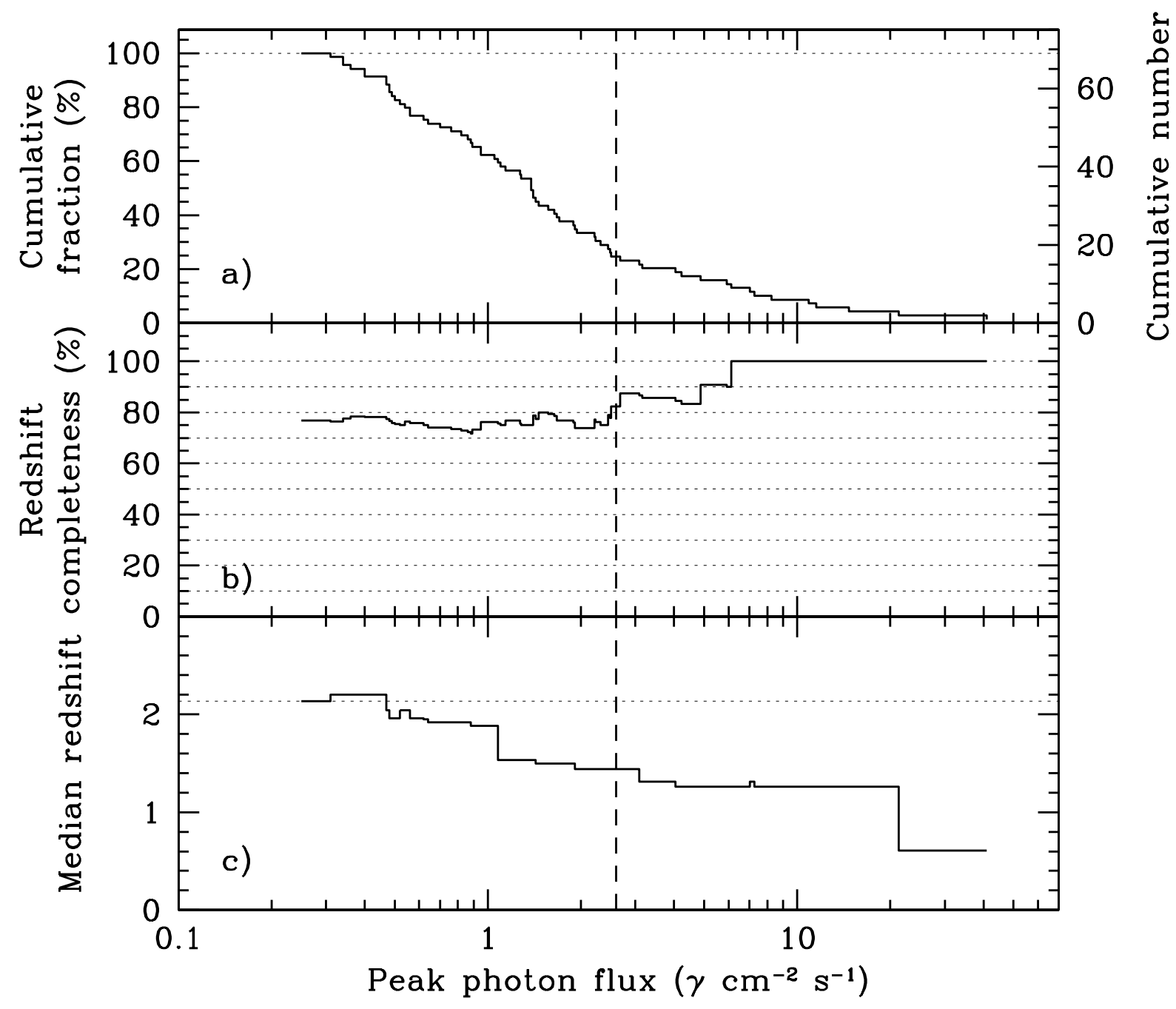

Fig. 10.- Redshift and BAT 15-150 keV peak photon flux. The vertical dashed line indicates the peak photon flux limit adopted by Salvaterra et al. (2012). (a) The cumulative distribution of peak photon fluxes of the TOUGH bursts. (b) The redshift completeness fraction of the TOUGH sample bursts as a function of the limiting peak photon flux. The redshift completeness is constant around $75 \%$ below $\approx 3 \gamma \mathrm{cm}^{-2} \mathrm{~s}^{-1}$. In order to significantly increase the completeness fraction, one would have to discard at least $>75 \%$ of all bursts. (c) The median redshift of TOUGH GRBs with known redshift brighter than the corresponding peak flux. Fainter GRBs are on average at higher redshift. 


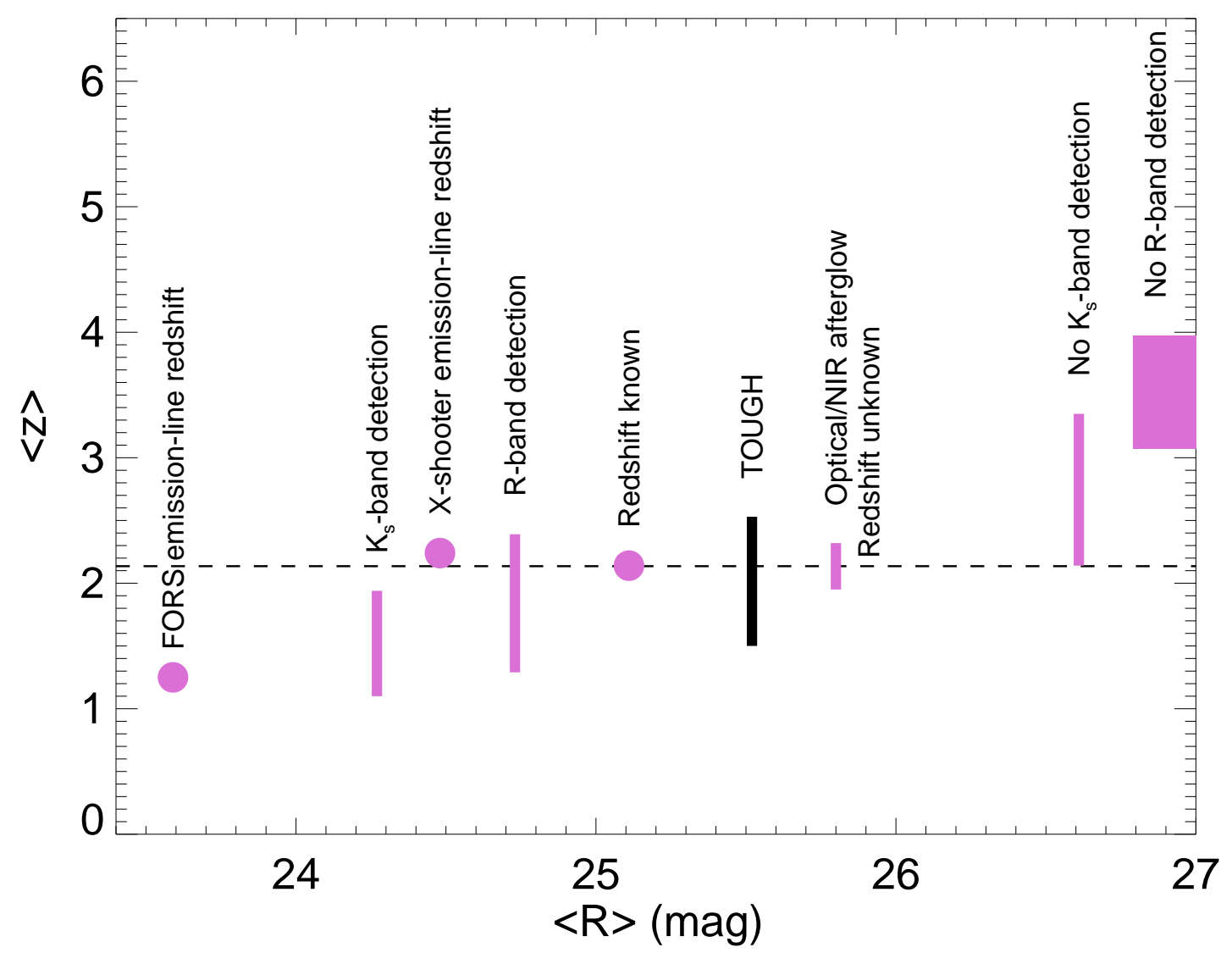

Fig. 11. - Median redshift (with a range of uncertainty where the median is not uniquely determined) against median $R$-band host magnitude for various subsets of our sample. In the case of those without an $R$-band detection, the set has been placed at $R \approx 27$. The figure broadly illustrates that the fainter hosts are typically at higher redshift, characteristic of our magnitude limit. The subset of hosts without redshift has a median $R \approx 25.9$ which suggests a median redshift above 2 if it follows the same trend. 

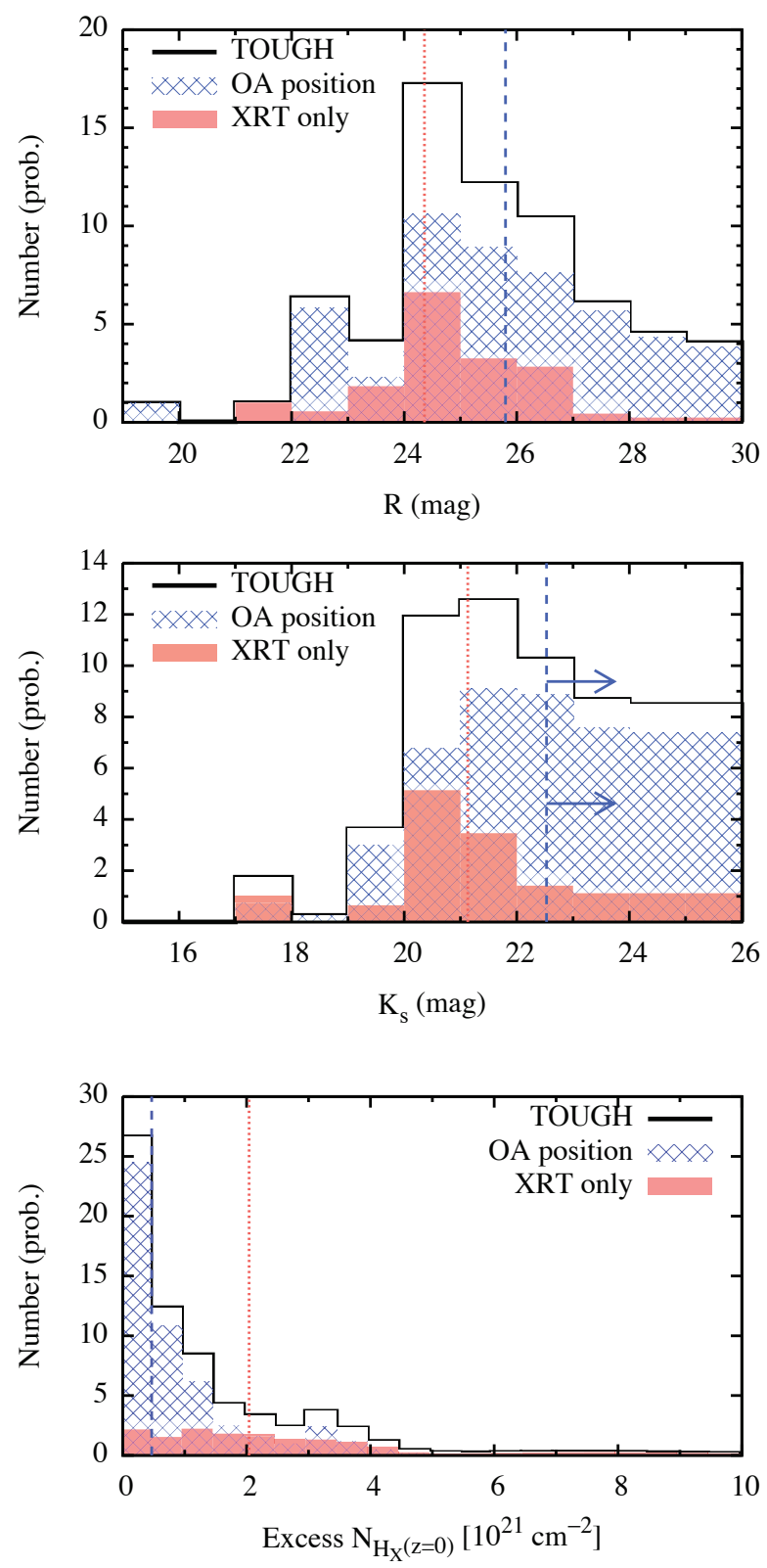

Fig. 12.- Distributions of host galaxy $R$ - (top) and $K_{s}$-band (middle) magnitudes as well as X-ray absorptions in excess of Galactic, determined at $z=0$ (bottom) for the TOUGH sample (open histogram). Subsets of galaxies selected with a localization from an optical/NIR afterglow (OA) and a localization from Swift/XRT only are shown as hatched and solid histograms, respectively. The corresponding median values (or upper limit to the median as indicated by arrows) are shown as dashed or dotted lines. The histograms were created from the sum of the normalized probability distributions for each object derived from their uncertainties or upper limits. For the galaxy magnitudes the upper limit probabilities were evenly distributed in magnitude space from the upper limit to a lower cutoff value: $R=30$ and $K_{s}=26$. While the height of the histogram at low values depends on this choice, the histogram area does not. 
Table 1. Sample Selection Criteria

\begin{tabular}{lll}
\hline \hline Criterion No. & \multicolumn{1}{c}{ Short Description } & \multicolumn{1}{c}{ Type of Constraint } \\
\hline 1 & Onboard Swift/BAT trigger & GRB properties \\
2 & Long GRBs: duration $T_{90}>2 \mathrm{~s}$ & GRB properties \\
3 & X-ray afterglow position available within $12 \mathrm{hr}$ & GRB properties, visibility, and slight biasing \\
4 & Galactic $A_{V} \leqslant 0.5 \mathrm{mag}$ & Visibility \\
5 & Sun distance $>55 \mathrm{deg}$ & Visibility \\
6 & Not near bright stars & Visibility \\
7 & 2005 Mar $1-2007$ Aug 10 & VLT sample \\
8 & $-70^{\circ}<\delta<+27^{\circ}(\mathrm{J} 2000.0)$ & VLT sample \\
9 & Localization accuracy better than $2^{\prime \prime}$ & Slight biasing \\
\hline
\end{tabular}


Table 2. The TOUGH Host Galaxy Sample

\begin{tabular}{|c|c|c|c|c|c|c|c|c|c|c|}
\hline $\begin{array}{l}\text { GRB } \\
(1)\end{array}$ & $\begin{array}{c}\text { Trigger No. } \\
(2)\end{array}$ & $\begin{array}{c}\text { R.A. } \\
\text { (J2000.0) } \\
(3)\end{array}$ & $\begin{array}{c}\text { Decl. } \\
(\mathrm{J} 2000.0) \\
(4)\end{array}$ & $\begin{array}{c}\text { Error } \\
\left({ }^{\prime \prime}\right) \\
(5)\end{array}$ & $\begin{array}{c}\text { Band } \\
(6)\end{array}$ & $\begin{array}{c}\text { Reference } \\
\text { (7) }\end{array}$ & $\begin{array}{c}A_{V} \\
(\mathrm{mag}) \\
(8)\end{array}$ & $\begin{array}{l}t_{\mathrm{XRT}} \\
(\mathrm{s}) \\
(9)\end{array}$ & $\begin{array}{c}T_{90} \\
(\mathrm{~s}) \\
(10)\end{array}$ & $\begin{array}{c}\text { Peak Flux } \\
\left(\gamma \mathrm{cm}^{-2} \mathrm{~s}^{-1}\right) \\
(11)\end{array}$ \\
\hline 050315 & 111063 & $20: 25: 54.14$ & $-42: 36: 01.5$ & 1.4 & $r$ & 1 & 0.16 & 84 & 95.6 & 1.89 \\
\hline 050318 & 111529 & 03:18:51.09 & $-46: 23: 45.8$ & 1.4 & $U$ & 2 & 0.05 & 3277 & 40.0 & 3.16 \\
\hline 050401 & 113120 & $16: 31: 28.80$ & $+02: 11: 14.5$ & 3.5 & $V$ & 3 & 0.22 & 124 & 33.3 & 10.90 \\
\hline 050406 & 113872 & 02:17:52.09 & $-50: 11: 17.1$ & 1.9 & $U$ & 4 & 0.07 & 106 & 4.8 & 0.36 \\
\hline 050416A & 114753 & $12: 33: 54.64$ & $+21: 03: 26.8$ & 1.4 & UVW2 & 5 & 0.10 & 78 & 6.6 & 4.88 \\
\hline 050502B & 116116 & $09: 30: 10.13$ & $+16: 59: 48.0$ & 1.4 & $R$ & 6 & 0.10 & 63 & 16.6 & 1.40 \\
\hline $050525 \mathrm{~A}$ & 130088 & $18: 32: 32.67$ & $+26: 20: 21.6$ & 1.5 & UVW2 & 7 & 0.32 & 125 & 8.8 & 41.00 \\
\hline 050714B & 145994 & $11: 18: 47.71$ & $-15: 32: 49.0$ & 1.5 & $\mathrm{X}$ & 8 & 0.18 & 151 & 46.9 & 0.52 \\
\hline 050726 & 147788 & $13: 20: 11.96$ & $-32: 03: 51.6$ & 1.4 & $V$ & 9 & 0.21 & 132 & 49.7 & 1.38 \\
\hline 050730 & 148225 & 14:08:17.18 & $-03: 46: 18.1$ & 1.4 & $B$ & 10,11 & 0.17 & 133 & 145.1 & 0.56 \\
\hline 050801 & 148522 & $13: 36: 35.32$ & $-21: 55: 42.7$ & 1.5 & UVW2 & 12 & 0.32 & 61 & 19.4 & 1.46 \\
\hline 050803 & 148833 & $23: 22: 37.85$ & $+05: 47: 08.5$ & 1.4 & $\mathrm{X}$ & 13 & 0.25 & 152 & 88.1 & 0.95 \\
\hline 050819 & 151131 & $23: 55: 01.62$ & $+24: 51: 39.0$ & 1.5 & $\mathrm{X}$ & 14 & 0.41 & 141 & 37.7 & 0.40 \\
\hline 050820A & 151207 & $22: 29: 38.12$ & $+19: 33: 37.0$ & 1.4 & UVW1 & 15 & 0.15 & 80 & 26.0 & 2.45 \\
\hline 050822 & 151486 & $03: 24: 27.18$ & $-46: 02: 00.0$ & 1.4 & $\mathrm{X}$ & 16 & 0.05 & 96 & 104.1 & 2.23 \\
\hline 050824 & 151905 & $00: 48: 56.21$ & $+22: 36: 33.1$ & 1.5 & UVW2 & 17 & 0.12 & 6089 & 24.8 & 0.49 \\
\hline 050904 & 153514 & $00: 54: 50.84$ & $+14: 05: 09.3$ & 3.5 & $I$ & 18 & 0.20 & 161 & 181.7 & 0.64 \\
\hline 050908 & 154112 & $01: 21: 50.70$ & $-12: 57: 18.0$ & 1.5 & $V$ & 19 & 0.08 & 106 & 18.3 & 0.70 \\
\hline 050915A & 155242 & $05: 26: 44.81$ & $-28: 00: 59.4$ & 1.4 & $H$ & 20 & 0.09 & 87 & 53.4 & 0.76 \\
\hline 050922B & 156434 & $00: 23: 13.37$ & $-05: 36: 16.7$ & 1.7 & $\mathrm{X}$ & 21 & 0.12 & 342 & 156.3 & 1.10 \\
\hline $050922 \mathrm{C}$ & 156467 & 21:09:33.08 & $-08: 45: 30.2$ & 1.4 & UVM2 & 15 & 0.34 & 108 & 4.5 & 7.04 \\
\hline 051001 & 157870 & $23: 23: 48.72$ & $-31: 31: 23.4$ & 1.7 & $\mathrm{X}$ & 22 & 0.05 & 192 & 190.6 & 0.48 \\
\hline 051006 & 158593 & $07: 23: 14.14$ & $+09: 30: 20.0$ & 1.5 & $\mathrm{X}$ & 23 & 0.22 & 113 & 26.0 & 1.70 \\
\hline 051016B & 159994 & $08: 48: 27.85$ & $+13: 39: 20.4$ & 1.4 & UVW2 & 24 & 0.12 & 75 & 4.0 & 1.28 \\
\hline 051117B & 164279 & 05:40:43.38 & $-19: 16: 27.2$ & 1.7 & $\mathrm{X}$ & 25 & 0.18 & 135 & 9.0 & 0.50 \\
\hline 060115 & 177408 & 03:36:08.32 & $+17: 20: 43.1$ & 1.4 & $R$ & 26 & 0.44 & 113 & 122.2 & 0.86 \\
\hline 060218 & 191157 & 03:21:39.69 & $+16: 52: 01.6$ & 1.4 & UVW2 & 27 & 0.47 & 153 & 2100.0 & 0.25 \\
\hline 060306 & 200638 & $02: 44: 22.88$ & $-02: 08: 54.7$ & 1.4 & $\mathrm{X}$ & 28 & 0.12 & 88 & 60.9 & 5.92 \\
\hline 060522 & 211117 & $21: 31: 44.89$ & $+02: 53: 10.1$ & 1.4 & $R$ & 29 & 0.18 & 142 & 69.1 & 0.54 \\
\hline 060526 & 211957 & $15: 31: 18.33$ & $+00: 17: 05.4$ & 1.4 & $B$ & 30,31 & 0.22 & 73 & 275.2 & 1.67 \\
\hline 060604 & 213486 & $22: 28: 55.04$ & $-10: 54: 56.1$ & 1.4 & UVW1 & 32 & 0.14 & 109 & 96.0 & 0.34 \\
\hline 060605 & 213630 & $21: 28: 37.31$ & $-06: 03: 30.7$ & 1.4 & $B$ & 33 & 0.16 & 93 & 539.1 & 0.47 \\
\hline 060607A & 213823 & $21: 58: 50.41$ & $-22: 29: 47.0$ & 1.4 & $U$ & 34 & 0.10 & 65 & 103.0 & 1.40 \\
\hline 060614 & 214805 & 21:23:32.12 & $-53: 01: 36.6$ & 1.4 & UVW2 & 35 & 0.07 & 91 & 109.2 & 11.50 \\
\hline 060707 & 217704 & $23: 48: 19.07$ & $-17: 54: 18.0$ & 1.7 & $B$ & 36 & 0.07 & 122 & 66.7 & 1.08 \\
\hline 060708 & 217805 & $00: 31: 13.80$ & $-33: 45: 32.6$ & 1.4 & UVW1 & 9 & 0.04 & 62 & 10.0 & 1.91 \\
\hline 060714 & 219101 & $15: 11: 26.46$ & $-06: 33: 58.8$ & 1.4 & $B$ & 37 & 0.26 & 99 & 116.0 & 1.27 \\
\hline 060719 & 220020 & 01:13:43.70 & $-48: 22: 50.6$ & 1.4 & $K$ & 38 & 0.03 & 77 & 66.9 & 2.21 \\
\hline 060729 & 221755 & $06: 21: 31.79$ & $-62: 22: 12.5$ & 1.4 & UVW2 & 39 & 0.18 & 124 & 113.0 & 1.14 \\
\hline 060805A & 222683 & $14: 43: 43.47$ & $+12: 35: 11.2$ & 1.6 & $\mathrm{X}$ & 40 & 0.08 & 93 & 4.9 & 0.31 \\
\hline 060814 & 224552 & $14: 45: 21.36$ & $+20: 35: 09.2$ & 1.4 & $K$ & 41 & 0.13 & 72 & 144.9 & 7.26 \\
\hline 060908 & 228581 & $02: 07: 18.42$ & $+00: 20: 32.2$ & 1.4 & UVW1 & 9 & 0.10 & 72 & 18.8 & 3.09 \\
\hline 060912A & 229185 & 00:21:08.13 & $+20: 58: 18.5$ & 1.4 & UWM2 & 9 & 0.17 & 109 & 5.0 & 8.27 \\
\hline 060919 & 230115 & $18: 27: 41.80$ & $-51: 00: 52.1$ & 1.7 & $\mathrm{X}$ & 42 & 0.24 & 87 & 9.0 & 2.31 \\
\hline
\end{tabular}


Table 2-Continued

\begin{tabular}{|c|c|c|c|c|c|c|c|c|c|c|}
\hline GRB & $\begin{array}{c}\text { Trigger No. } \\
(2)\end{array}$ & $\begin{array}{c}\text { R.A. } \\
\text { (J2000.0) } \\
(3)\end{array}$ & $\begin{array}{c}\text { Decl. } \\
(\mathrm{J} 2000.0) \\
(4)\end{array}$ & $\begin{array}{l}\text { Error } \\
\left({ }^{\prime \prime}\right) \\
(5)\end{array}$ & $\begin{array}{l}\text { Band } \\
\text { (6) }\end{array}$ & $\begin{array}{c}\text { Reference } \\
\text { (7) }\end{array}$ & $\begin{array}{c}A_{V} \\
(\mathrm{mag}) \\
(8)\end{array}$ & $\begin{array}{c}t_{\mathrm{XRT}} \\
(\mathrm{s}) \\
(9)\end{array}$ & $\begin{array}{l}T_{90} \\
(\mathrm{~s}) \\
(10)\end{array}$ & $\begin{array}{c}\text { Peak Flux } \\
\left(\gamma \mathrm{cm}^{-2} \mathrm{~s}^{-1}\right) \\
(11)\end{array}$ \\
\hline 060923A & 230662 & $16: 58: 28.14$ & $+12: 21: 37.9$ & 1.5 & $K$ & 43 & 0.20 & 81 & 51.5 & 1.38 \\
\hline 060923B & 230702 & $15: 52: 46.70$ & $-30: 54: 13.7$ & 1.8 & $\mathrm{X}$ & 44 & 0.49 & 114 & 8.9 & 1.43 \\
\hline 060923C & 230711 & $23: 04: 28.28$ & $+03: 55: 28.1$ & 3.5 & $J$ & 45 & 0.21 & 203 & 67.4 & 0.89 \\
\hline 060927 & 231362 & $21: 58: 12.05$ & $+05: 21: 49.0$ & 1.6 & $I$ & 46 & 0.21 & 65 & 22.4 & 2.68 \\
\hline 061004 & 232339 & $06: 31: 10.81$ & $-45: 54: 24.2$ & 1.4 & $\mathrm{X}$ & 47 & 0.16 & 60 & 6.3 & 2.48 \\
\hline 061007 & 232683 & 03:05:19.60 & $-50: 30: 02.4$ & 1.4 & UVW2 & 48 & 0.07 & 80 & 75.7 & 14.70 \\
\hline 061021 & 234905 & 09:40:36.12 & $-21: 57: 05.2$ & 1.4 & UVW2 & 9 & 0.19 & 78 & 43.8 & 6.12 \\
\hline 061110A & 238108 & $22: 25: 09.89$ & $-02: 15: 30.4$ & 1.6 & $R$ & 49 & 0.30 & 69 & 44.5 & 0.48 \\
\hline 061110B & 238174 & $21: 35: 40.46$ & $+06: 52: 32.9$ & 1.7 & $R$ & 50 & 0.13 & 3195 & 132.8 & 0.47 \\
\hline 061121 & 239899 & 09:48:54.59 & $-13: 11: 42.1$ & 1.4 & UVW2 & 51 & 0.15 & 55 & 81.2 & 21.30 \\
\hline 070103 & 254532 & 23:30:13.80 & $+26: 52: 34.4$ & 1.5 & $\mathrm{X}$ & 52 & 0.22 & 69 & 18.4 & 1.05 \\
\hline 070110 & 255445 & 00:03:39.38 & $-52: 58: 28.1$ & 1.4 & $U$ & 53 & 0.05 & 93 & 79.7 & 0.62 \\
\hline 070129 & 258408 & 02:28:00.98 & $+11: 41: 03.4$ & 1.4 & $R$ & 54 & 0.46 & 134 & 459.1 & 0.56 \\
\hline 070224 & 261880 & 11:56:06.57 & $-13: 19: 48.8$ & 1.6 & $R$ & 55 & 0.19 & 143 & 48.0 & 0.34 \\
\hline 070306 & 263361 & 09:52:23.29 & $+10: 28: 55.5$ & 1.4 & $H$ & 56 & 0.09 & 153 & 209.2 & 4.04 \\
\hline 070318 & 271019 & 03:13:56.77 & $-42: 56: 47.3$ & 1.4 & UVW2 & 57 & 0.06 & 64 & 131.5 & 1.64 \\
\hline 070328 & 272773 & $04: 20: 27.68$ & $-34: 04: 00.7$ & 1.4 & $\mathrm{X}$ & 58 & 0.12 & 88 & 72.1 & 4.22 \\
\hline 070330 & 273180 & $17: 58: 10.20$ & $-63: 47: 34.4$ & 1.5 & $V$ & 59 & 0.21 & 68 & 6.6 & 0.88 \\
\hline 070419B & 276212 & 21:02:49.77 & $-31: 15: 48.7$ & 1.5 & $V$ & 60 & 0.30 & 81 & 238.1 & 1.38 \\
\hline 070506 & 278693 & 23:08:52.31 & $+10: 43: 20.8$ & 1.8 & $B$ & 61 & 0.13 & 127 & 4.3 & 0.95 \\
\hline 070611 & 282003 & 00:07:58.12 & $-29: 45: 20.4$ & 1.8 & UVW1 & 62 & 0.04 & 3300 & 13.2 & 0.82 \\
\hline 070621 & 282808 & $21: 35: 10.08$ & $-24: 49: 03.1$ & 1.4 & $\mathrm{X}$ & 63 & 0.16 & 111 & 33.3 & 2.50 \\
\hline 070721B & 285654 & $02: 12: 32.93$ & $-02: 11: 39.7$ & 1.5 & $V$ & 64 & 0.10 & 92 & 336.9 & 1.57 \\
\hline 070802 & 286809 & $02: 27: 35.78$ & $-55: 31: 39.5$ & 1.5 & $B$ & 65 & 0.09 & 138 & 15.8 & 0.40 \\
\hline 070808 & 287260 & 00:27:03.36 & $+01: 10: 34.4$ & 1.5 & $\mathrm{X}$ & 66 & 0.08 & 114 & 58.4 & 1.94 \\
\hline 050412 & 114485 & $12: 04: 25.28$ & $-01: 12: 00.6$ & 3.7 & $\mathrm{X}$ & 67 & 0.07 & 99 & 26.5 & 0.49 \\
\hline 050603 & 131560 & 02:39:56.89 & $-25: 10: 54.9$ & 1.5 & $V$ & 68 & 0.09 & 39024 & 22.0 & 15.30 \\
\hline 060117 & 177666 & $21: 51: 36.23$ & $-59: 58: 39.3$ & 1.5 & $R$ & 69 & 0.12 & $\ldots$ & 16.9 & 48.80 \\
\hline 060223A & 192059 & 03:40:49.56 & $-17: 07: 49.7$ & 1.5 & $V$ & 9 & 0.39 & 86 & 11.3 & 1.35 \\
\hline 060313 & 201487 & $04: 26: 28.46$ & $-10: 50: 41.2$ & 1.4 & UVW2 & 70 & 0.23 & 79 & 0.7 & 10.80 \\
\hline 060505 & 208654 & 22:07:03.32 & $-27: 48: 53.0$ & 2.0 & $B$ & 71 & 0.07 & 51744 & 4.0 & 2.65 \\
\hline $060602 \mathrm{~A}$ & 213180 & 09:58:16.28 & $+00: 18: 14.4$ & 5.2 & $R$ & 72 & 0.08 & 155520 & 74.7 & 0.56 \\
\hline 070721A & 285653 & 00:12:39.13 & $-28: 33: 00.9$ & 1.6 & $\mathrm{X}$ & 73 & 0.05 & 86 & 3.4 & 0.68 \\
\hline 070810A & 287364 & $12: 39: 51.24$ & $+10: 45: 03.2$ & 1.4 & $V$ & 74 & 0.07 & 88 & 9.0 & 1.89 \\
\hline 080207 & 302728 & $13: 50: 02.93$ & $+07: 30: 07.9$ & 1.4 & $\mathrm{X}$ & 75 & 0.08 & 124 & 340.0 & 1.00 \\
\hline
\end{tabular}

Note. - Column 1: GRB name; Column 2: Swift trigger number; Columns 3-5: X-ray position and error Goad et al. 2007; Evans et al. 2009); the position of GRB 060117 is taken from Jelínek et al. (2006); Column 6: bluest optical/NIR afterglow detection, excluding white and unfiltered measurements; "X" indicates no optical/NIR detection; Column 7: reference for the afterglow detection; Column 8: Galactic $V$-band absorption (Schlegel et al. 1998); Column 9: time to start of the XRT observation; Column 10: observed gamma-ray duration $T_{90}$ (Sakamoto et al. 2011); Column 11: peak photon flux over $1 \mathrm{~s}$ timescale (15-150 keV; Sakamoto et al. 2011). The horizontal line separates the objects in the TOUGH sample from 10 extra systems that were observed within our program.

References. (1) Berger et al. (2005); (2) Still et al. (2005); (3) Kamble et al. (2009); (4) Schadv et al. (2006); (5) Holland et al. (2007); (6) Afonso et al. (2011); (7) Blustin et al. (2006); (8) Levan et al. (2005); (9) Oates et al. (2009); (10) Pandev et al. (2006); (11) Perri et al. (2007); (12) de Pasquale et al. (2007); (13) Band et al. (2005); (14) Kennea et al. (2005); (15) Schady et al. (2010); (16) 
Blustin et al. (2005a); (17) Schady et al. (2007b); (18) Tagliaferri et al. (2005); (19) de Pasquale et al. (2005); (20) Bloom \& Alatalo (2005); (21) Norris et al. (2005); (22) Moretti et al. (2005); (23) Morris et al. (2005); (24) Blustin et al. (2005b); (25) Beardmore et al. (2005); (26) Yanagisawa et al. (2006); (27) Campana et al. (2006); (28) Angelini et al. (2006); (29) D'Avanzo et al. (2006); (30) Dai et al. (2007); (31) Thöne et al. (2010); (32) Blustin \& Page (2006); (33) Ferrero et al. (2009); (34) Ziaeepour et al. (2008); (35) Mangano et al. (2007); (36) Schady \& Moretti (2006); (37) Asfandvarov et al. (2006); (38) Malesani et al. (2006); (39) Grupe et al. (2007); (40) Ziaeepour et al. (2006b); (41) Levan et al. (2006b); (42) Guidorzi et al. (2006); (43) Tanvir et al. (2008); (44) Stamatikos et al. (2006); (45) Covino et al. (2006); (46) Ruiz-Velasco et al. (2007); (47) Ziaeepour et al. (2006a); (48) Schady et al. (2007a); (49) Chen et al. (2006); (50) Melandri et al. (2006); (51) Page et al. (2007); (52) Sakamoto et al. (2007); (53) Troja et al. (2007); (54) Malesani et al. (2007); (55) Thoene et al. (2007a); (56) Jaunsen et al. (2008); (57) Chester et al. (2008); (58) Markwardt et al. (2007); (59) Kuin et al. (2007); (60) de Ugarte Postigo et al. (2007); (61) Landsman \& Pagani (2007); (62) Landsman et al. (2007); (63) Sbarufatti et al. (2007); (64) de Pasquale \& Ziaeepour (2007); (65) Elíasdóttir et al. (2009); (66) Cummings et al. (2007); (67) Mineo et al. (2007); (68) Grupe et al. (2006); (69) Jelínek et al. (2006); (70) Roming et al. (2006); (71) Xu et al. (2009); (72) Jensen et al. (2006); (73) Ziaeepour et al. (2007); (74) Chester et al. (2007); (75) Racusin et al. (2008). 
Table 3. Catalog of Survey Results

\begin{tabular}{|c|c|c|c|c|c|c|c|}
\hline $\begin{array}{l}\text { GRB } \\
(1)\end{array}$ & $\begin{array}{c}\text { R.A. } \\
\text { (J2000.0) } \\
(2)\end{array}$ & $\begin{array}{c}\text { Decl. } \\
\text { (J2000.0) } \\
(3)\end{array}$ & $\begin{array}{c}R \\
(\mathrm{mag}) \\
(4)\end{array}$ & $\begin{array}{c}K_{\mathrm{S}} \\
(\mathrm{mag}) \\
(5)\end{array}$ & $\begin{array}{c}\text { Redshift } \\
\text { (6) }\end{array}$ & $\begin{array}{c}\text { Notes } \\
(7)\end{array}$ & $\begin{array}{c}\text { Reference } \\
(8)\end{array}$ \\
\hline 050315 & $20: 25: 54.189$ & $-42: 36: 02.16$ & $24.43 \pm 0.15$ & $21.89 \pm 0.36$ & 1.9500 & $\mathrm{~A}$ & 1 \\
\hline 050318 & $\ldots$ & $\ldots$ & $>26.79$ & $>22.31$ & 1.4436 & $\mathrm{~A}$ & 1 \\
\hline 050401 & $16: 31: 28.794$ & $+02: 11: 14.33$ & $26.15 \pm 0.31$ & $>21.73$ & 2.8983 & $\mathrm{~A}^{*}$ & 2,3 \\
\hline 050406 & $02: 17: 52.224$ & $-50: 11: 14.97$ & $26.61 \pm 0.34$ & $>21.25$ & 2.70 & $\mathrm{P}$ & 4 \\
\hline $050416 \mathrm{~A}$ & $12: 33: 54.602$ & $+21: 03: 26.58$ & $23.13 \pm 0.03$ & $20.36 \pm 0.20$ & 0.6528 & $\mathrm{E}$ & 1,5 \\
\hline 050502B & $\ldots$ & $\ldots$ & $>26.79$ & $>21.81$ & 5.2 & $\mathrm{P}$ & 6 \\
\hline 050525A & $18: 32: 32.590$ & $+26: 20: 22.60$ & $25.95 \pm 0.34$ & $>21.23$ & 0.606 & $\mathrm{AE}$ & 7 \\
\hline 050714B & $11: 18: 47.717$ & $-15: 32: 49.01$ & $25.45 \pm 0.20$ & $20.39 \pm 0.17$ & $\ldots$ & $\ldots$ & $\ldots$ \\
\hline 050726 & $\ldots$ & $\ldots$ & $>26.14$ & $>21.28$ & $\ldots$ & $\ldots$ & $\ldots$ \\
\hline 050730 & $\cdots$ & $\ldots$ & $>27.20$ & $>21.36$ & 3.96855 & $\mathrm{~A}^{*}$ & $8,9,3,10$ \\
\hline 050801 & $\ldots$ & $\ldots$ & $>26.78$ & $>21.70$ & 1.38 & $\mathrm{P}$ & 11,12 \\
\hline 050803 & $23: 22: 37.850$ & $+05: 47: 08.90$ & $26.18 \pm 0.33$ & $>21.50$ & $\ldots$ & $\ldots$ & $\ldots$ \\
\hline 050819 & $23: 55: 01.598$ & $+24: 51: 38.16$ & $24.10 \pm 0.09$ & $\ldots$ & 2.5043 & $\mathrm{E}$ & 13 \\
\hline 050820A & $22: 29: 38.114$ & $+19: 33: 36.61$ & $24.83 \pm 0.18$ & $>21.18$ & 2.61469 & $\mathrm{~A}^{*}$ & 14,15 \\
\hline 050822 & $03: 24: 27.214$ & $-46: 01: 59.43$ & $24.19 \pm 0.08$ & $20.78 \pm 0.20$ & 1.434 & $\mathrm{E}$ & 16 \\
\hline 050824 & $00: 48: 56.151$ & $+22: 36: 32.80$ & $24.23 \pm 0.16$ & $>21.41$ & 0.8278 & $\mathrm{AE}$ & 17,3 \\
\hline 050904 & $\ldots$ & $\ldots$ & $\ldots$ & $\ldots$ & 6.295 & A & 18 \\
\hline 050908 & $01: 21: 50.730$ & $-12: 57: 17.30$ & $27.76 \pm 0.45$ & $>21.91$ & 3.3467 & $\mathrm{~A}^{*}$ & 3,19 \\
\hline 050915A & $05: 26: 44.843$ & $-28: 00: 59.62$ & $24.56 \pm 0.16$ & $20.69 \pm 0.24$ & 2.5273 & $\mathrm{E}$ & 13 \\
\hline 050922B & $00: 23: 13.345$ & $-05: 36: 18.31$ & $26.18 \pm 0.30$ & $>22.17$ & $\ldots$ & $\ldots$ & $\ldots$ \\
\hline $050922 \mathrm{C}$ & $\ldots$ & $\ldots$ & $>26.34$ & $>21.47$ & 2.1992 & $\mathrm{~A}^{*}$ & 20,21 \\
\hline 051001 & $23: 23: 48.800$ & $-31: 31: 23.45$ & $24.36 \pm 0.13$ & $21.13 \pm 0.35$ & 2.4296 & $\mathrm{E}$ & 13 \\
\hline 051006 & $07: 23: 14.109$ & $+09: 30: 19.96$ & $22.99 \pm 0.07$ & $\ldots$ & 1.059 & $\mathrm{E}$ & 16 \\
\hline 051016B & $08: 48: 27.844$ & $+13: 39: 20.07$ & $22.64 \pm 0.03$ & $20.04 \pm 0.20$ & 0.9364 & $\mathrm{E}$ & 22 \\
\hline 051117B & $05: 40: 43.287$ & $-19: 16: 26.31$ & $21.06 \pm 0.02$ & $17.75 \pm 0.05$ & 0.481 & $\mathrm{E}$ & 16 \\
\hline 060115 & $03: 36: 08.355$ & $+17: 20: 42.65$ & $27.14 \pm 0.53$ & $>21.27$ & 3.5328 & $\mathrm{~A}^{*}$ & 3 \\
\hline 060218 & $03: 21: 39.683$ & $+16: 52: 02.09$ & $19.98 \pm 0.01$ & $17.94 \pm 0.09$ & 0.03351 & $\mathrm{E}$ & $23,24,25,26,27$ \\
\hline 060306 & $02: 44: 22.930$ & $-02: 08: 54.55$ & $24.09 \pm 0.08$ & $19.95 \pm 0.17$ & $\ldots$ & $\ldots$ & $\ldots$ \\
\hline 060522 & $\ldots$ & $\ldots$ & $>26.64$ & $>23.15$ & 5.110 & $\mathrm{~A}$ & 28 \\
\hline 060526 & $\ldots$ & $\ldots$ & $>27.02$ & $>22.11$ & 3.2213 & A & $29,20,30$ \\
\hline 060604 & $22: 28: 55.029$ & $-10: 54: 55.72$ & $25.52 \pm 0.18$ & $>21.37$ & 2.1357 & $\mathrm{AE}$ & 3,13 \\
\hline 060605 & $\ldots$ & $\cdots$ & $>26.54$ & $>21.12$ & 3.773 & A & 31 \\
\hline 060607A & $\ldots$ & $\ldots$ & $>27.92$ & $>21.86$ & 3.0749 & $\mathrm{~A}$ & 15 \\
\hline 060614 & $21: 23: 32.103$ & $-53: 01: 36.20$ & $22.53 \pm 0.06$ & $20.36 \pm 0.20$ & 0.125 & $\mathrm{E}$ & 32,33 \\
\hline 060707 & $23: 48: 19.061$ & $-17: 54: 17.72$ & $24.86 \pm 0.06$ & $>19.71$ & 3.4240 & $\mathrm{~A}$ & 20,3 \\
\hline 060708 & $00: 31: 13.748$ & $-33: 45: 32.73$ & $26.76 \pm 0.28$ & $>21.04$ & 1.92 & $\mathrm{P}$ & 11 \\
\hline 060714 & $15: 11: 26.436$ & $-06: 33: 58.24$ & $26.45 \pm 0.28$ & $>21.40$ & 2.7108 & $\mathrm{AE}^{*}$ & 20,3 \\
\hline 060719 & $01: 13: 43.706$ & $-48: 22: 51.31$ & $24.62 \pm 0.12$ & $21.25 \pm 0.27$ & 1.5320 & $\mathrm{E}$ & 13 \\
\hline 060729 & $06: 21: 31.794$ & $-62: 22: 12.53$ & $23.71 \pm 0.04$ & $21.20 \pm 0.31$ & 0.5428 & A & 3 \\
\hline $060805 \mathrm{~A}$ & $14: 43: 43.381$ & $+12: 35: 10.28$ & $23.48 \pm 0.03$ & $20.69 \pm 0.15$ & $\ldots$ & $\ldots$ & $\ldots$ \\
\hline 060805A & $14: 43: 43.488$ & $+12: 35: 12.56$ & $25.11 \pm 0.14$ & $21.66 \pm 0.38$ & $\ldots$ & $\ldots$ & $\ldots$ \\
\hline 060814 & $14: 45: 21.347$ & $+20: 35: 10.72$ & $22.85 \pm 0.11$ & $20.06 \pm 0.10$ & 1.9229 & $\mathrm{E}$ & $16,13,34$ \\
\hline 060908 & $02: 07: 18.406$ & $+00: 20: 31.55$ & $25.53 \pm 0.18$ & $22.53 \pm 0.43$ & 1.8836 & $\mathrm{AE}$ & 35,3 \\
\hline 060912A & $00: 21: 08.127$ & $+20: 58: 17.66$ & $22.68 \pm 0.04$ & $19.86 \pm 0.09$ & 0.937 & $\mathrm{E}$ & 36 \\
\hline
\end{tabular}


Table 3-Continued

\begin{tabular}{|c|c|c|c|c|c|c|c|}
\hline GRB & $\begin{array}{c}\text { R.A. } \\
\text { (J2000.0) } \\
(2)\end{array}$ & $\begin{array}{c}\text { Decl. } \\
\text { (J2000.0) } \\
(3)\end{array}$ & $\begin{array}{c}R \\
(\mathrm{mag}) \\
(4)\end{array}$ & $\begin{array}{c}K_{\mathrm{s}} \\
(\mathrm{mag}) \\
(5)\end{array}$ & $\begin{array}{c}\text { Redshift } \\
\text { (6) }\end{array}$ & Notes & $\begin{array}{c}\text { Reference } \\
\text { (8) }\end{array}$ \\
\hline 060919 & $18: 27: 41.790$ & $-51: 00: 50.94$ & $25.79 \pm 0.26$ & $21.41 \pm 0.38$ & $\cdots$ & $\cdots$ & $\ldots$ \\
\hline 060923A & $16: 58: 28.175$ & $+12: 21: 38.96$ & $26.07 \pm 0.24$ & $>21.25$ & $\cdots$ & $\cdots$ & $\cdots$ \\
\hline 060923B & $15: 52: 46.566$ & $-30: 54: 14.57$ & $24.34 \pm 0.16$ & $20.39 \pm 0.19$ & $\cdots$ & $\cdots$ & $\cdots$ \\
\hline 060923B & $15: 52: 46.583$ & $-30: 54: 12.70$ & $24.40 \pm 0.16$ & $20.59 \pm 0.20$ & $\cdots$ & $\cdots$ & $\cdots$ \\
\hline 060923C & $23: 04: 28.266$ & $+03: 55: 29.29$ & $25.45 \pm 0.18$ & $21.73 \pm 0.34$ & $\ldots$ & $\ldots$ & $\ldots$ \\
\hline 060927 & $\ldots$ & $\ldots$ & $\ldots$ & $>21.76$ & 5.4636 & A & 37,3 \\
\hline 061004 & $\ldots$ & $\ldots$ & $>25.76$ & $>21.24$ & $\ldots$ & $\ldots$ & $\ldots$ \\
\hline 061007 & 03:05:19.587 & $-50: 30: 02.43$ & $24.40 \pm 0.17$ & $21.06 \pm 0.37$ & 1.2622 & $\mathrm{AE}$ & 38,3 \\
\hline 061021 & 09:40:36.124 & $-21: 57: 05.05$ & $24.44 \pm 0.06$ & $>21.14$ & 0.3463 & $\mathrm{AE}$ & 16,3 \\
\hline 061110A & $22: 25: 09.843$ & $-02: 15: 31.12$ & $25.65 \pm 0.26$ & $>21.41$ & 0.7578 & $\mathrm{E}$ & 3 \\
\hline 061110B & $21: 35: 40.400$ & $+06: 52: 33.91$ & $26.04 \pm 0.29$ & $>21.24$ & 3.4344 & $\mathrm{~A}^{*}$ & 3 \\
\hline 061121 & $09: 48: 54.564$ & $-13: 11: 43.09$ & $22.75 \pm 0.03$ & $20.14 \pm 0.19$ & 1.3145 & $\mathrm{~A}^{*}$ & 3 \\
\hline 070103 & 23:30:13.793 & $+26: 52: 35.32$ & $24.18 \pm 0.14$ & $21.07 \pm 0.36$ & 2.6208 & $\mathrm{E}$ & 13 \\
\hline 070110 & 00:03:39.268 & $-52: 58: 27.12$ & $25.02 \pm 0.11$ & $22.41 \pm 0.50$ & 2.3521 & $\mathrm{AE}^{*}$ & 3 \\
\hline 070129 & 02:28:00.915 & $+11: 41: 04.56$ & $24.39 \pm 0.12$ & $21.01 \pm 0.18$ & 2.3384 & $\mathrm{E}$ & 13 \\
\hline 070224 & $11: 56: 06.637$ & $-13: 19: 48.33$ & $25.96 \pm 0.31$ & $>21.20$ & $\ldots$ & $\ldots$ & $\ldots$ \\
\hline 070306 & 09:52:23.310 & $+10: 28: 55.28$ & $22.90 \pm 0.08$ & $19.70 \pm 0.10$ & 1.49594 & $\mathrm{E}$ & 39,16 \\
\hline 070318 & $03: 13: 56.800$ & $-42: 56: 46.25$ & $24.43 \pm 0.11$ & $>21.04$ & 0.8397 & A & 3,40 \\
\hline 070328 & $04: 20: 27.615$ & $-34: 04: 00.63$ & $24.43 \pm 0.13$ & $>20.98$ & $\ldots$ & $\ldots$ & $\ldots$ \\
\hline 070330 & $\ldots$ & $\ldots$ & $>26.15$ & $>21.19$ & $\ldots$ & $\ldots$ & $\ldots$ \\
\hline 070419B & $21: 02: 49.801$ & $-31: 15: 48.82$ & $25.23 \pm 0.20$ & $>21.22$ & 1.9591 & $\mathrm{E}$ & 13 \\
\hline 070506 & 23:08:52.392 & $+10: 43: 21.00$ & $26.10 \pm 0.22$ & $>21.36$ & 2.3090 & A & 3 \\
\hline 070611 & $\ldots$ & $\ldots$ & $>27.09$ & $>21.75$ & 2.0394 & $\mathrm{~A}$ & 3 \\
\hline 070621 & 21:35:10.061 & $-24: 49: 02.18$ & $25.77 \pm 0.23$ & $>21.51$ & $\cdots$ & $\cdots$ & $\cdots$ \\
\hline 070721B & $02: 12: 32.950$ & $-02: 11: 40.80$ & $27.53 \pm 0.44$ & $>22.38$ & 3.6298 & $\mathrm{~A}^{*}$ & 3 \\
\hline 070802 & $02: 27: 35.722$ & $-55: 31: 38.76$ & $25.11 \pm 0.21$ & $21.27 \pm 0.41$ & 2.4541 & $A^{*}$ & 41,3 \\
\hline 070808 & 00:27:03.310 & $+01: 10: 35.81$ & $26.71 \pm 0.33$ & $21.77 \pm 0.37$ & $\cdots$ & $\cdots$ & $\ldots$ \\
\hline 050412 & $12: 04: 25.460$ & $-01: 12: 00.05$ & $25.55 \pm 0.19$ & $>21.40$ & $\cdots$ & $\cdots$ & $\cdots$ \\
\hline 050603 & $\ldots$ & $\ldots$ & $>26.61$ & $>22.66$ & $\ldots$ & $\ldots$ & $\ldots$ \\
\hline 060117 & $\ldots$ & $\ldots$ & $>26.28$ & $\ldots$ & $\ldots$ & $\ldots$ & $\ldots$ \\
\hline 060223A & $\ldots$ & $\ldots$ & $>26.25$ & $>21.24$ & 4.406 & A & 28 \\
\hline 060313 & $\ldots$ & $\ldots$ & $\ldots$ & $>21.13$ & $\ldots$ & $\ldots$ & $\ldots$ \\
\hline 060505 & 22:07:03.500 & $-27: 48: 55.57$ & $17.90 \pm 0.00$ & $16.22 \pm 0.01$ & 0.0889 & $\mathrm{E}$ & $42,43,44$ \\
\hline $060602 \mathrm{~A}$ & $09: 58: 16.750$ & $+00: 18: 12.56$ & $23.88 \pm 0.08$ & $20.56 \pm 0.27$ & 0.787 & $\mathrm{E}$ & 45 \\
\hline 070721A & $00: 12: 39.048$ & $-28: 33: 00.14$ & $25.16 \pm 0.17$ & $\ldots$ & $\ldots$ & $\cdots$ & $\ldots$ \\
\hline 070721A & $00: 12: 39.139$ & $-28: 33: 00.98$ & $23.14 \pm 0.15$ & $\ldots$ & $\ldots$ & $\ldots$ & $\ldots$ \\
\hline 070810A & $\ldots$ & $\ldots$ & $>26.73$ & $>21.93$ & 2.17 & A & 46 \\
\hline 080207 & $13: 50: 02.997$ & $+07: 30: 07.44$ & $25.84 \pm 0.18$ & $\ldots$ & 2.0858 & $\mathrm{E}$ & 13 \\
\hline
\end{tabular}

Note. - Column1: GRB name; Columns 2 and 3: host coordinates; Column 4: $R$-band Vega magnitude; Column 5: $K_{\mathrm{S}}$-band Vega magnitude; Column 6: redshift; numbers in boldface were determined from our program; Column 7: source of the redshift: (A)bsorption features in the afterglow spectrum (*, with finestructure transitions), (E)mission lines from the host, or (P)hotometric redshift of the afterglow; Column 8: redshift reference. The horizontal line separates the bursts in the TOUGH sample from 10 extra hosts that were observed within our program. 
References. (1) Berger et al. (2005); (2) Watson et al. (2006); (3) Fvnbo et al. (2009); (4) Schadv et al. (2006); (5) Soderberg et al. (2007); (6) Afonso et al. (2011); (7) Della Valle et al. (2006b); (8) Chen et al. (2005); (9) Starling et al. (2005); (10) D'Elia et al. (2007); (11) Oates et al. (2009); (12) de Pasquale et al. (2007); (13) Krühler et al. (2012); (14) Prochaska et al. (2007); (15) Fox et al. (2008); (16) Jakobsson et al. (2012); (17) Sollerman et al. (2007); (18) Kawai et al. (2006); (19) Prochaska et al. (2005); (20) Jakobsson et al. (2006a); (21) Piranomonte et al. (2008); (22) Soderberg et al. (2005); (23) Mirabal et al. (2006); (24) Sollerman et al. (2006); (25) Modiaz et al. (2006); (26) Pian et al. (2006); (27) Wiersema et al. (2007); (28) Charv et al. (2007); (29) Berger \& Gladders (2006); (30) Thöne et al. (2010); (31) Ferrero et al. (2009); (32) Gal-Yam et al. (2006); (33) Della Valle et al. (2006a); (34) Salvaterra et al. (2012); (35) Milvang-Jensen et al. (2012); (36) Levan et al. (2007); (37) Ruiz-Velasco et al. (2007); (38) Osip et al. (2006); (39) Jaunsen et al. (2008); (40) Chen et al. (2007); (41) Elíasdóttir et al. (2009); (42) Ofek et al. (2007); (43) Fynbo et al. (2006); (44) Thöne et al. (2008); (45) Jakobsson et al. (2007); (46) Thoene et al. (2007b). 
Table 4. Median Properties of TOUGH and TOUGH Subsamples

\begin{tabular}{|c|c|c|c|c|c|}
\hline $\begin{array}{l}\text { Sample } \\
\text { (1) }\end{array}$ & $\begin{array}{c}R \\
(2)\end{array}$ & $\begin{array}{l}K_{s} \\
(3)\end{array}$ & $\begin{array}{c}\langle z\rangle_{\text {low }} \\
(4)\end{array}$ & $\begin{array}{c}\text { Median } z \\
\quad(5)\end{array}$ & $\begin{array}{c}\langle z\rangle_{\text {high }} \\
\quad(6)\end{array}$ \\
\hline TOUGH & $25.52 \pm 0.23$ & $>22.53$ & 1.50 & $\ldots$ & 2.53 \\
\hline FORS & $23.59 \pm 0.51$ & $20.78 \pm 1.30$ & $\cdots$ & $1.25 \pm 0.29$ & $\cdots$ \\
\hline X-shooter & $24.48 \pm 0.15$ & $21.13 \pm 0.27$ & $\cdots$ & $2.24 \pm 0.15$ & $\ldots$ \\
\hline $\mathrm{OA}$ & $25.80 \pm 0.31$ & $>22.53$ & 1.95 & $\ldots$ & 2.32 \\
\hline XRT & $24.36 \pm 0.40$ & $21.13 \pm 0.29$ & $\ldots$ & $\ldots$ & $\ldots$ \\
\hline$z$ & $25.11 \pm 0.31$ & $>22.53$ & $\ldots$ & $2.14 \pm 0.18$ & $\cdots$ \\
\hline No $z$ & $25.88 \pm 0.24$ & $>21.77$ & $\ldots$ & $\ldots$ & $\ldots$ \\
\hline$R$ & $24.73 \pm 0.21$ & $22.15 \pm 0.69$ & 1.29 & $\ldots$ & 2.39 \\
\hline No $R$ & $\ldots$ & $\ldots$ & 3.07 & $\ldots$ & 3.97 \\
\hline$K_{s}$ & $24.27 \pm 0.28$ & $20.74 \pm 0.16$ & 1.10 & $\ldots$ & 1.94 \\
\hline No $K_{s}$ & $26.61 \pm 0.43$ & $\ldots$ & 2.14 & $\ldots$ & 3.35 \\
\hline
\end{tabular}

Note. - Column 1: TOUGH sample or subsample; Column 2: median $R$ magnitude of subsample; Column 3: median $K_{s}$ magnitude of subsample; Column 4: minimum median redshift of subsample, assuming all unknown redshifts are less than the minimum redshift; Column 5: median redshift; Column 6: maximum median redshift of subsample, assuming all unknown redshifts are larger than the maximum redshift.

Errors in the median are estimated as $1.4826 \times \mathrm{MAD} / \sqrt{N-1}$, where MAD is the median absolute deviation, $N$ is the number of systems used to determine the median and 1.4826 is a scale factor that applies to Gaussian distributions. The subsamples are the following: FORS: the six well-defined FORS emissionline redshifts, i.e., excluding the dropout redshifts (GRBs 050822, 051006, 051117B, 060908, 061021, and 070306); X-shooter: the nine new X-shooter redshifts; OA: systems with an optical/NIR afterglow; XRT: systems with no optical/NIR afterglow; $z$ : systems with a known redshift; No $z$ : systems without a known redshift; $R$ : systems with a measured $R$ magnitude; No $R$ : systems without a measured $R$ magnitude (including the high-redshift systems GRB 050904 and GRB 060927 which were not observed); $K_{s}$ : systems with a measured $K_{s}$ magnitude; No $K_{s}$ : systems without a measured $K_{s}$ magnitude (including the high-redshift GRB 050904 but excluding GRB 050819 and GRB 051006 which were not observed). 